\title{
Congenital skeletal malformations induced by lamb da-cyhalothrin, dibenzalacetone and heptanone derivatives in embryos of albino mice
}

El Sayed Aly Mohammed Metwally ${ }^{1}$, Mohammed Abdel-fattah Desheesh ${ }^{3}$, Doaa Mohammed Abd El-kawy Elmasry ${ }^{3}$, Mahmoud Morshedy Farg ${ }^{3}$, Hamada Mahmoud Youssef ${ }^{2}$, Rasha M. Elshinety*1.

${ }^{* 1}$ Department of Anatomy and Embryology, Faculty of Medicine, Alexandria University, Alexandria, Egypt

${ }^{2}$ Chief Researher , Agriculture Research Center - Plant Protection Station, Alexandria, Egypt,

${ }^{3}$ Department of Pesticide chemistry and technology, Faculty of Agriculture, Alexandria University, Alexandria, Egypt.

\section{ABSTRACT}

Background: Insecticides such as lambda-cyhalothrin(LCT), dibenzalacetone (DBA) and heptanone HD are teratogenic substances. Skeleton is one of the organs sensitive to teratogens during the period of organogenesis.

Material and methods: This study was conducted on fifty adult female albino mice, divided randomly into four groups: Group I (control group, $n=5$ received corn oil orally in adose of $10 \mathrm{mg} / \mathrm{kg}$ body weight), group II (LCT group, $\mathrm{n}=10$ received LCT (nano $8 \%$ )10, $20 \mathrm{mg} / \mathrm{kg}$ body weight for each subgroup), group III (DBA group, $n=20$, received DBA (main) 10, $20 \mathrm{mg} / \mathrm{kg}$ body weight and DBA (nano 8\%)) 10,20 mg / kg body weight for each subgroup) and group IV (HD group, $\mathrm{n}=15$, received HD (main) $10 \mathrm{mg} / \mathrm{kg}$ body weight and HD (nano 8\%)) 10,50mg $/ \mathrm{kg}$ body weight for each subgroup),The calculated treatment dissolved in $1 \mathrm{ml}$ of corn oil by orogastric tube was given to the pregnant mice during the first week of pregnancy (1st, 3rd and 5th day).The delivered live and dead pups were examined externally, then double stained and prepared for skeletal examination by dissecting microscope.

Results: All experimental groups showed various anomalies that can be categorized as the following: 1) presence of dead pups and resorption sites 2) macroscopic anomalies in the form of anencephaly, extended forelimb, cranioshisis, macroglossia, lowset ears, lage eye bulge, half body, amelia, internal rotation of forelimb and encephalocele. 3) skeletal anomalies by double staining of fetal skeleton showed incomplete ossification of nasal and interparietal bones, open arch of atlas, incomplete ossification of vertebrae, supernumerary ribs, wavy ribs, incomplete ossification of ribs and sternebrae, incomplete ossification of sternum, incomplete ossification of 5 th metacarpal and terminal phalangal bones and incomplete ossification of tarsal, and phalangal bones.

Conclusion: Lambda-cyhalothrin, dibenzalacetone and derivatives of heptanone result in numbers of congenital gross and skeletal abnormalities that indicate their teratogenic effect.

KEY WORDS: Lambada, Congenital, Skeletal, Dibenzalacetone, Heptanone, Teratogenic.

Corresponding Author: Rasha El-shinety, MD, PhD, Ass. Prof. of Anatomy \& Embryology, Alexandria Faculty of Medicine, Director of Project Management Unit, Alexandria University, Egypt. Mobile: 01061230422 E-Mail: rashaelshinety@yahoo.com

\section{Access this Article online}

Quick Response code

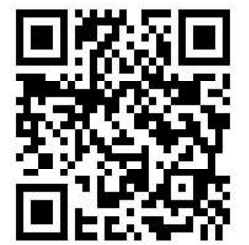

DOI: $10.16965 /$ ijar.2021.109
Journal Information

International Journal of Anatomy and Research

ISSN (E) 2321-4287 | ISSN (P) 2321-8967

https://www.ijmhr.org/ijar.htm

DOI-Prefix: https://dx.doi.org/10.16965/ijar

\section{Article Information}

Received: 18 Jan 2021

Peer Review: 19 Jan 2021

Revised: None
Accepted: 22 Feb 2021

Published (O): 05 Mar 2021

Published (P): 05 Mar 2021 


\section{INTRODUCTION}

Synthetic pyrethroids are a group of insectisides that are commonly used both in agriculture and as domestic insectisides. Lambda-cyhalothrin (LCT), is a synthetic pyrethroid, that has been widely used in many countries including Egypt in the last two decades to control agricultural pests, veterinary and human insects. Previous studies have shown that excessive exposure to pyrthroids can cause liver damage with a severity associated with the dose and duration of exposure [1].

Preparation and properties of lambda cyhalothrin / polyurethane drug - loaded nanoemulsions were carried out by adding iso phorone diisocyanate $(3.67 \mathrm{~g})$, polyether diol (4.0gm), castor oil $(1.38 \mathrm{gm})$ and 2 drops of dibutyltin dilaurate into a $100 \mathrm{ml}$ three necked round bottom flask equipped with an electric mechanical stirrer and a reflux condenser and the mixture was heated to $80^{\circ} \mathrm{C}$ with the stirring speed of $300 \mathrm{rpm}$. researchers determined the characterization of the prepared nanoemulsion for their chemical composition, colloidal and thermal property, solid content, surface tension and controlled release behavior [2-4].

Excessive uncontrolled use of these prepartions results in serious health problems, which is contributed to the unavoidable transportation of these chemicals and their degradation products to non-target species and fostering environmental and human health concerns $[1,5]$.

The poor water solubility of these compounds and the use of different organic solvents to remove them may cause more contamination of the atmosphere by the solvent itself $[6,7]$.

Skeleton is one of the organs that is most sensitive to teratogens during the period of organogenesis, insecticides like lambdacyhalothrin, dibenzalacetone and heptanone derivatives are widely used that neecssites the evaluation of their teratogenicity[8, 9].

The toxicological properties of dibenzalacetone and heptanone derivative have not been fully investigated so far.
Aim of the study: The objective of this study is to investigate the teratogenic effects of LCT, DBA and HD during the organogenetic period on albino mice embryos.

\section{MATERIALS AND METHODS}

\section{Chemicals and reagents:}

1. Lambda-Cyhalothrin (LCT) 92\%TC (Insecticide Pyrethroid), was obtained from Egyptchem International for Agrochemicals, Alexandria, Egypt.

2. Dibenzalacetone (DBA) (laboratory preparation)

3. Heptanone -triene (laboratory preparation)

The main and nano-encapsulated compounds have been used as the following table 1 :

Table 1: Main and nanoparticles of Lambda cyhalothrin, Dibenzalacetone and Heptanone derivatives.

\begin{tabular}{ccc}
\hline Compound & Main & Nano 8\% \\
\hline Lambda - cyhalothrin (LCT) & - & $10 \mathrm{mg}$ \\
& - & $20 \mathrm{mg}$ \\
Dibenzalacetone (DBA) & $10 \mathrm{mg}$ & $10 \mathrm{mg}$ \\
Heptanone derivatives HD & $25 \mathrm{mg}$ & $25 \mathrm{mg}$ \\
& $10 \mathrm{mg}$ & $10 \mathrm{mg}$ \\
\hline
\end{tabular}

Synthesis of Nanoencapsulated synthetic pesticides: Melt-dispersion process has been used to prepare the nanoparticles[10]. Specific polyethylene glycol (PEG) 6000 components were heated at $65^{\circ} \mathrm{C}$ separately. Various parts of LCT, DBA and HD were combined and stirred gently to these molten PEG. At room temperature, the mixtures were allowed to cool. Completely grounded in a mortar, the solidified mass was sieved using a 200 mesh sieve. The nanoparticles was prepared from the main compound according to the following equation

Nano $(8 \%)=4 \times 100 \div 46$

Size characterization of nanoparticles: Transmission Electron Microscopy (TEM) determined the morphology of the most potent nano-encapsulated form. Nanozetasizer (Malvern) was used to analyze the size and distribution of nanoparticles[11].

Animals and treatments: This study was conducted on fifty pregnant female albino mice (Musmus culus var.albus); their mean weight 
was $30 \pm 2$ grams, around 7-9 weeks old. The mice have been obtained from Animal House, Department of Physiology, El Mowasah, Faculty of Medicine, University of Alexandria.

The LD 50 of LCT is $24 \mathrm{mg} / \mathrm{kg}$ body weight [12], the DBA is $300 \mathrm{mg} / \mathrm{kg}$ body weight [13] and the HD is $>700 \mathrm{mg} / \mathrm{kg}$ body weight. Sub-lethal dosages were tested at the first week of gestation on pregnant mice (1st, 3rd and 5 th day), the pregnant mice with vaginal plug were randomly divided into four groups each group was given the calculated dose of the treatment dissolved in $1 \mathrm{ml}$ corn oil by orogastric tube for 3 days (table 2).

Group I: (control group), five mice received corn oil in a dose of $10 \mathrm{mg} / \mathrm{kg}$ body weight.

Group II: treated group with nanoparticles the group consists of 20 mices equally divided into 4 subroups and received the nano- encapsulated compounds as in table I

Group III: treated group consists of 15 mices divided into 3 subgroups and received the compounds as in table I

Termination of pregnancy was done though abdominal section of all gravid females at the $2 \mathbf{1}^{\text {st }}$ day of gestation: Examination of the live and dead pups by naked eye for gross congenital anomalies was done, some pups were kept in bouin solution [14].

The live pups were weighted, examined externally and after death doubled stained (Alician blue, Alzerin red) [15] and examined by dissecting microscope (stereoscope) (Experimental Embryology Laboratory, Department of Anatomy and Embryology, El Mowasah, Faculty of Medicine, Alexandria) and photomicrographs were taken.

scarification of the adult mice and uterine horns were examined for resorption sites: Data analysis was fed to the computer and analyzed using version 20.0 of the IBM SPSS software package. (Armonk, NY: IBM Corp) $[16,17]$.

Results were expressed as mean \pm standard errors (mean \pm SEM). All analysis was carried out with GraphPad Prism 6.0 for Windows (GraphPad Software, San Diego, CA). Significant differences between treatment effects were determined by one-way ANOVA, followed by Tukey's post hoc test for multiple comparisons, while correlation analyses were performed by spearman test. Statistical significance was set at $p<0.05$.

\section{RESULTS}

No significant statistical difference in maternal body weight was observed between different study groups as shown in (Table 2, Figure 1). In group III b (DBA main $25 \mathrm{mg} / \mathrm{kg}$ ) and group IV c (HD Nano $50 \mathrm{mg} / \mathrm{kg}$ ), the overall number of dead pups was significantly increased compared to the control group (Table 3 , Figure 2).

Resorption sites (Figure 3) were significantly increased in groups Ila (LCT Nano $10 \mathrm{mg} / \mathrm{kg}$ ), IIId (DBA Nano $25 \mathrm{mg} / \mathrm{kg}$ ) and IV b (HD Nano $10 \mathrm{mg} / \mathrm{kg}$ ) compared to the control group. (Table 3 , Figure 3).

Gross congenital anomalies [19] (table 4, figures 4-13): Anencephally (absent skull vault and brain) was significantly increased in groups IIIb (DBA main $25 \mathrm{mg} / \mathrm{kg}$ ), group IVa (HD main $10 \mathrm{mg} / \mathrm{kg}$ ) and group IVc (HD nano $50 \mathrm{mg} / \mathrm{kg}$ ) compared to the control group (Table 4 , Figures 4,5 ).

Extended fore-limb in group IIIa (DBA main $10 \mathrm{mg} / \mathrm{kg}$ ) was significantly increased compared to the control group (Table 4 , Figures 4,5 ).

Cranioschisis was evident in group IVc (HD nano $50 \mathrm{mg} / \mathrm{kg}$ ) compared to the control group (Table 4 , Figures 6,7 ).

Macroglossia was markedly increased in groups IIla (DBA main $10 \mathrm{mg} / \mathrm{kg}$ ) and group Ivc (HD nano $50 \mathrm{mg} / \mathrm{kg}$ ) compared to the control group (Table 4 , Figures 8,9 ).

The low set ears were significantely increased groups Illa (DBA main 10mg kg), IIlc (DBA nano 10mg / $\mathrm{kg}$ ), Illd (DBA nano $25 \mathrm{mg} / \mathrm{kg}$ ) and grouplvc (HD nano $50 \mathrm{mg} / \mathrm{kg}$ ) as compared to the control group (Table 4 , Figures 8, 9).

Large eye bulge was significantly increased in groups IIb (LCT nano $20 \mathrm{mg} / \mathrm{kg}$ ) and group IVc (HD nano $50 \mathrm{mg} / \mathrm{kg}$ ) compared to the control group (Table 4 , Figures 8,9).

Half body was also significantly increased in groups IIla (DBA main $10 \mathrm{mg} / \mathrm{kg}$ ), group Iva 
Table 2: Maternal body weight distribution among various study groups.

\begin{tabular}{|c|c|c|c|c|c|c|c|c|c|c|}
\hline \multirow[b]{2}{*}{$\begin{array}{l}\text { Maternal body } \\
\text { weight (gm) }\end{array}$} & \multirow[b]{2}{*}{$\begin{array}{l}\text { Group I } \\
\text { (control) (N } \\
=5)\end{array}$} & \multicolumn{2}{|c|}{ Group II (LCT) } & \multicolumn{4}{|c|}{ Group III (DBA) } & \multicolumn{3}{|c|}{ Group IV [18] } \\
\hline & & $\begin{array}{c}\text { Ila } \\
\text { (Nano } \\
10 \mathrm{mg} / \mathrm{kg}) \\
(\mathrm{N}=5)\end{array}$ & $\begin{array}{c}\text { Ilb } \\
\text { (Nano } \\
20 \mathrm{mg} / \mathrm{kg}) \\
(\mathrm{N}=5)\end{array}$ & $\begin{array}{c}\text { Illa } \\
\text { (main } \\
10 \mathrm{mg} / \mathrm{kg}) \\
(\mathrm{N}=5)\end{array}$ & $\begin{array}{c}\text { IIIb } \\
\text { (main } \\
25 \mathrm{mg} / \mathrm{kg}) \\
(\mathrm{N}=5)\end{array}$ & $\begin{array}{c}\text { Illc } \\
\text { (Nano } \\
10 \mathrm{mg} / \mathrm{kg}) \\
(\mathrm{N}=5)\end{array}$ & $\begin{array}{c}\text { Illd } \\
\text { (Nano } \\
25 \mathrm{mg} / \mathrm{kg}) \\
(\mathrm{N}=5)\end{array}$ & $\begin{array}{c}\text { Iva } \\
\text { (main } \\
10 \mathrm{mg} / \mathrm{kg}) \\
(\mathrm{N}=5)\end{array}$ & $\begin{array}{c}\text { IVb } \\
\text { (Nano } \\
10 \mathrm{mg} / \mathrm{kg}) \\
(\mathrm{N}=5)\end{array}$ & $\begin{array}{c}\text { IVc } \\
\text { (Nano } \\
50 \mathrm{mg} / \mathrm{kg}) \\
(\mathrm{N}=5)\end{array}$ \\
\hline Min. - Max. & $32.0-35.0$ & $25.0-30.0$ & $25.0-31.0$ & $27.0-32.0$ & $28.0-32.0$ & $27.0-31.0$ & $25.0-33.0$ & $24.0-30.0$ & $24.0-30.0$ & $25.0-29.0$ \\
\hline Mean \pm SD. & $34.0 \pm 1.22$ & $28.0 \pm 1.87$ & $27.80 \pm 2.28$ & $29.60 \pm 1.82$ & $30.20 \pm 1.79$ & $29.20 \pm 1.64$ & $28.40 \pm 3.85$ & $26.80 \pm 2.28$ & $26.60 \pm 2.70$ & $27.0 \pm 1.58$ \\
\hline Median & 34 & 28 & 27 & 30 & 30 & 30 & 27 & 26 & 25 & 27 \\
\hline$p_{0}$ & & $0.004^{*}$ & $0.003^{*}$ & 0.083 & 0.205 & $0.042^{*}$ & $0.009^{*}$ & $<0.001^{*}$ & $<0.001^{*}$ & $<0.001^{*}$ \\
\hline $\begin{array}{l}\text { Sig bet. } \\
\text { subgroups }\end{array}$ & & \multicolumn{2}{|c|}{$\mathrm{p}_{1}=1.000$} & & $\begin{array}{l}p_{2}=1.000, p_{3}= \\
p 5=0.999, p 6=\end{array}$ & $\begin{array}{l}.000, p_{4}=0.997, \\
.952, p 7=1.000\end{array}$ & & \multicolumn{3}{|c|}{$p_{8}=1.000, p_{9}=1.000, p_{10}=1.000$} \\
\hline
\end{tabular}

Pairwise comparison bet. each 2 groups was done using Post Hoc Test (Tukey)for ANOVA test

$p_{0}: p$ value for comparing between control and each other subgroups

$\mathrm{p}_{1}$ : $\mathrm{p}$ value for comparing between Ila and IIb

$\mathrm{p}_{2}$ : $p$ value for comparing between IIIa and IIIb

$p_{3}: p$ value for comparing between IIIa and IIIc

$p_{4}: p$ value for comparing between IIIa and IIId

$\mathrm{p}_{5}: \mathrm{p}$ value for comparing between IIIb and IIIc

$p_{6}: p$ value for comparing between IIIb and IIId

$p_{7}: p$ value for comparing between IIIc and IIId

$\mathrm{p}_{8}: \mathrm{p}$ value for comparing between IVa and IVb

$p_{9}: p$ value for comparing between IVa and IVc

$\mathrm{p}_{10}: \mathrm{p}$ value for comparing between IVb and IVc

*: Statistically significant at $p \leq 0.05$

Table 3: Distribution of live and dead pups among study groups.

\begin{tabular}{|c|c|c|c|c|c|c|c|c|c|c|}
\hline \multirow{3}{*}{$\begin{array}{l}\text { Number of } \\
\text { implantation }\end{array}$} & \multirow{3}{*}{$\begin{array}{c}\text { Group I } \\
\text { (control) }\end{array}$} & \multicolumn{2}{|c|}{ Group II (LCT) } & \multicolumn{4}{|c|}{ Group III (DBA) } & \multicolumn{3}{|c|}{ Group IV [18] } \\
\hline & & Ila & Ilb & Illa & IIIlb & IIII & IIId & Iva & IVb & IVc \\
\hline & & $\begin{array}{c}\text { (Nano } \\
10 \mathrm{mg} / \mathrm{kg} \text { ) }\end{array}$ & $\begin{array}{c}\text { (Nano } \\
20 \mathrm{mg} / \mathrm{kg} \text { ) }\end{array}$ & $\begin{array}{c}\text { (main } \\
10 \mathrm{mg} / \mathrm{kg})\end{array}$ & $\begin{array}{c}\text { (main } \\
25 \mathrm{mg} / \mathrm{kg})\end{array}$ & $\begin{array}{c}\text { (Nano } \\
10 \mathrm{mg} / \mathrm{kg})\end{array}$ & $\begin{array}{c}\text { (Nano } \\
25 \mathrm{mg} / \mathrm{kg} \text { ) }\end{array}$ & $\begin{array}{c}\text { (main } \\
10 \mathrm{mg} / \mathrm{kg})\end{array}$ & $\begin{array}{c}\text { (Nano } \\
10 \mathrm{mg} / \mathrm{kg})\end{array}$ & $\begin{array}{c}\text { (Nano } \\
50 \mathrm{mg} / \mathrm{kg})\end{array}$ \\
\hline No. & 5 & 5 & 5 & 5 & 5 & 5 & 5 & 5 & 5 & 5 \\
\hline $\begin{array}{l}\text { Total no. of } \\
\text { implants }\end{array}$ & 43 & 42 & 39 & 28 & 34 & 31 & 32 & 24 & 42 & 36 \\
\hline $\begin{array}{l}\text { Total no. of live } \\
\text { fetuses }\end{array}$ & $43(100.0)$ & $35(83.3 \%)$ & 37 (94.9\%) & 20 (71.4\%) & 24 (70.6\%) & $31(100.0)$ & 25 (78.1\%) & $24(100.0)$ & $35(83.3 \%)$ & $24(66.7 \%)$ \\
\hline $\begin{array}{l}\text { Total no. of dead } \\
\text { fetuses }\end{array}$ & - & - & $2(5.1 \%)$ & $4(14.3 \%)$ & $6(17.6 \%)$ & - & $2(6.3 \%)$ & - & - & 7 (19.4\%) \\
\hline Resorption sites & - & $7(16.7 \%)$ & - & $4(14.3 \%)$ & $4(11.8 \%)$ & - & $5(15.6 \%)$ & - & $7(16.7 \%)$ & $5(13.9 \%)$ \\
\hline $\mathbf{p}_{\mathrm{c}}$ & & \multicolumn{2}{|c|}{0.068} & \multicolumn{4}{|c|}{$0.004^{*}$} & \multicolumn{3}{|c|}{$0.005^{*}$} \\
\hline Sig bet. subgroups & & \multicolumn{2}{|c|}{$\mathrm{p}_{1}=0.157$} & \multicolumn{4}{|c|}{$\begin{array}{c}p_{2}=1.000, p_{3}=0.002^{*}, p_{4}=0.624, \\
p 5=0.001^{*}, p 6=0.394, p 7=0.012^{*}\end{array}$} & \multicolumn{3}{|c|}{$p_{8}=0.042^{*}, p_{9}=0.004^{*}, p_{10}=0.008^{*}$} \\
\hline
\end{tabular}

Sig bet. subgroups $p_{1}=0.157 p_{2}=1.000, p_{3}=0.002^{*}, p_{4}=0.624, p_{5}=0.001^{*}, p_{6}=0.394, p_{7}=0.012^{*}$ $p_{8}=0.042^{*}, p_{9}=0.004^{*}, p_{10}=0.008^{*}$

$P_{c}: p$ value for comparing between control and each other groups

$\mathrm{p}_{1}$ : $\mathrm{p}$ value for comparing between Ila and IIb

$\mathrm{p}_{2}$ : $\mathrm{p}$ value for comparing between IIIa and IIIb

$p_{3}: p$ value for comparing between IIIa and IIIc

$\mathrm{p}_{4}: p$ value for comparing between IIIa and IIId

$\mathrm{p}_{5}$ : $\mathrm{p}$ value for comparing between IIIb and IIIc

$\mathrm{p}_{6}$ : $\mathrm{p}$ value for comparing between IIIb and IIId

$\mathrm{p}_{7}: p$ value for comparing between IIIc and IIId

$p_{8}: p$ value for comparing between IVa and IVb

$p_{g}: p$ value for comparing between IVa and IVc

$p_{10}: p$ value for comparing between IVb and IVc

*: Statistically significant at $p \leq 0.05$ 
Table 4: Gross congenital anomalies in mice pups among study groups.

\begin{tabular}{|c|c|c|c|c|c|c|c|c|c|c|}
\hline \multirow{3}{*}{$\begin{array}{c}\text { Gross congenital } \\
\text { abnormalities }\end{array}$} & \multirow{3}{*}{$\begin{array}{l}\text { Group I } \\
\text { (control) (n } \\
=43 \text { ) }\end{array}$} & \multicolumn{2}{|c|}{ Group II (LCT) $(n=81)$} & \multicolumn{4}{|c|}{ Group III (DBA) $(n=125)$} & \multicolumn{3}{|c|}{ Group IV [18] $(n=102)$} \\
\hline & & $\mathrm{Ila}(\mathrm{n}=\mathbf{4 2})$ & $\mathrm{Ilb}(\mathrm{n}=39)$ & $\mathrm{IIIa}(\mathrm{n}=28)$ & $\mathrm{IIIb}(\mathrm{n}=34)$ & $\mathrm{IIIc}(\mathrm{n}=\mathbf{3 1})$ & IIId(n = 32) & Iva $(n=24)$ & $\mathrm{IVb}(n=42)$ & $\operatorname{IVc}(n=36)$ \\
\hline & & $\begin{array}{c}\text { (Nano } \\
10 \mathrm{mg} / \mathrm{kg})\end{array}$ & $\begin{array}{c}\text { (Nano } \\
20 \mathrm{mg} / \mathrm{kg})\end{array}$ & $\begin{array}{c}\text { (main } \\
10 \mathrm{mg} / \mathrm{kg})\end{array}$ & $\begin{array}{c}\text { (main } \\
25 \mathrm{mg} / \mathrm{kg})\end{array}$ & $\begin{array}{c}\text { (Nano } \\
10 \mathrm{mg} / \mathrm{kg})\end{array}$ & $\begin{array}{c}\text { (Nano } \\
25 \mathrm{mg} / \mathrm{kg})\end{array}$ & $\begin{array}{c}\text { (main } \\
10 \mathrm{mg} / \mathrm{kg})\end{array}$ & $\begin{array}{c}\text { (Nano } \\
10 \mathrm{mg} / \mathrm{kg})\end{array}$ & $\begin{array}{c}\text { (Nano } \\
50 \mathrm{mg} / \mathrm{kg})\end{array}$ \\
\hline Anencephally & 1 & 2 & 3 & 2 & 4 & 3 & 3 & 4 & 1 & 5 \\
\hline $\mathbf{p}_{\mathrm{c}}$ & & \multicolumn{2}{|c|}{0.664} & \multicolumn{4}{|c|}{0.187} & \multicolumn{3}{|c|}{0.175} \\
\hline $\begin{array}{l}\text { Sig bet. } \\
\text { Subgroups }\end{array}$ & & \multicolumn{2}{|c|}{$\mathrm{p}_{1}=0.668$} & \multicolumn{4}{|c|}{$\begin{array}{c}p_{2}=0.681, p_{3}=1.000, p_{4}=1.000, p_{5}=1.000, p_{6}=1.000 \\
p_{7}=1.000\end{array}$} & \multicolumn{3}{|c|}{$\mathrm{p}_{8}=0.055, \mathrm{p}_{9}=1.000, \mathrm{p}_{10}=0.090$} \\
\hline $\begin{array}{l}\text { Extended } \\
\text { forelimb }\end{array}$ & 1 & 2 & 2 & 3 & 1 & - & 1 & 1 & - & 2 \\
\hline $\mathbf{p}_{\mathrm{c}}$ & & \multicolumn{2}{|c|}{0.658} & \multicolumn{4}{|c|}{1} & \multicolumn{3}{|c|}{1} \\
\hline $\begin{array}{l}\text { Sig bet. } \\
\text { Subgroups }\end{array}$ & & \multicolumn{2}{|c|}{$p_{1}=1.000$} & \multicolumn{4}{|c|}{$\begin{array}{c}p_{2}=0.320, p_{3}=0.101, p_{4}=0.331, p_{5}=1.000, p_{6}=1.000 \\
p_{7}=1.000\end{array}$} & \multicolumn{3}{|c|}{$p_{8}=0.364, p_{9}=1.000, p_{10}=0.210$} \\
\hline Cranioschisis & 0 & 0 & 0 & 0 & 0 & - & 0 & 0 & 0 & 1 \\
\hline $\mathbf{p}_{\mathrm{c}}$ & & \multicolumn{2}{|c|}{-} & \multicolumn{4}{|c|}{-} & \multicolumn{3}{|c|}{1} \\
\hline $\begin{array}{l}\text { Sig bet. } \\
\text { subgroups }\end{array}$ & & \multicolumn{2}{|c|}{-} & \multicolumn{4}{|c|}{-} & \multicolumn{3}{|c|}{$p_{9}=1.000, p_{10}=0.462$} \\
\hline Macroglossia & 0 & 1 & 1 & 2 & 0 & 1 & 1 & 0 & 1 & 2 \\
\hline $\mathbf{p}_{\mathrm{c}}$ & & \multicolumn{2}{|c|}{0.543} & \multicolumn{4}{|c|}{0.573} & \multicolumn{3}{|c|}{0.555} \\
\hline $\begin{array}{l}\text { Sig bet. } \\
\text { subgroups }\end{array}$ & & \multicolumn{2}{|c|}{$p_{1}=1000$} & $\mathrm{p}_{2}=0.200, \mathrm{p}_{3}$ & $\begin{aligned} 3=0.599, p_{4} & =0 \\
p_{7} & =1\end{aligned}$ & $\begin{array}{l}.594, p_{5}=0.47 \\
1.000\end{array}$ & $77, p_{6}=0.485$ & $\mathrm{p}_{8}=1.00$ & $0, p_{9}=0.512, p$ & ${ }_{10}=0.593$ \\
\hline Low set ear & 0 & - & - & 1 & - & 1 & 1 & - & - & 1 \\
\hline $\mathbf{p}_{\mathrm{c}}$ & & - & - & & 0.5 & 571 & & & 1 & \\
\hline $\begin{array}{l}\text { Sig bet. } \\
\text { subgroups }\end{array}$ & & - & - & $p_{2}=0.452, p_{3}$ & $\begin{aligned} 3=1.000, p_{4} & =1 \\
p_{7} & =1\end{aligned}$ & $\begin{array}{l}1.000, p_{5}=0.47 \\
1.000\end{array}$ & $77, p_{6}=0.485$ & $\mathrm{p}_{9}=$ & $=1.000, \mathrm{p}_{10}=0$ & 462 \\
\hline Large eye bulge & - & 1 & 2 & 1 & - & 1 & 1 & 1 & 1 & 2 \\
\hline $\mathbf{p}_{\mathrm{c}}$ & & 0.5 & 51 & & 0.5 & 571 & & & 0.319 & \\
\hline $\begin{array}{c}\text { Sig bet. } \\
\text { subgroups }\end{array}$ & & $\mathrm{p}_{1}=0$ & .606 & $p_{2}=0.452, p_{3}$ & $\begin{array}{r}3=1.000, p_{4}=1 \\
p_{7}=1\end{array}$ & $\begin{array}{l}1.000, p_{5}=0.47 \\
1.000\end{array}$ & $77, p_{6}=0.485$ & $\mathrm{p}_{8}=1.00$ & $0, p_{9}=1.000, p$ & ${ }_{10}=0.593$ \\
\hline Half body & - & - & - & 1 & - & - & - & 1 & - & 1 \\
\hline $\mathbf{p}_{\mathrm{c}}$ & & - & - & & & 1 & & & 1 & \\
\hline $\begin{array}{c}\text { Sig bet. } \\
\text { subgroups }\end{array}$ & & & & & ${ }_{2}=0.452, p_{3}=0$ & $0.475, p_{4}=0.46$ & & $\mathrm{p}_{8}=0.36$ & $4, p_{9}=1.000, p$ & $10=0.462$ \\
\hline Amelia & - & 1 & 2 & 1 & - & 2 & 1 & 1 & 1 & 1 \\
\hline $\mathbf{p}_{\mathrm{c}}$ & & 0.5 & 51 & & 0.5 & 573 & & & 0.37 & \\
\hline $\begin{array}{c}\text { Sig bet. } \\
\text { subgroups }\end{array}$ & & $\mathrm{p}_{1}=0$ & .606 & $p_{2}=0.452, p_{3}$ & $\begin{aligned} 3=1.000, p_{4} & =1 \\
p_{7} & =0\end{aligned}$ & $\begin{array}{l}1.000, p_{5}=0.22 \\
0.613\end{array}$ & $24, p_{6}=0.485$ & $\mathrm{p}_{8}=1.00$ & $0, p_{9}=1.000, p$ & $10=1.000$ \\
\hline $\begin{array}{l}\text { Internal rotation } \\
\text { of forelimb }\end{array}$ & - & 1 & 2 & 1 & - & 1 & 1 & 1 & 1 & 2 \\
\hline $\mathbf{p}_{\mathrm{c}}$ & & 0.5 & 551 & & 0.5 & 571 & & & 0.319 & \\
\hline $\begin{array}{c}\text { Sig bet. } \\
\text { Subgroups }\end{array}$ & & $\mathrm{p}_{1}=0$ & .606 & $\mathrm{p}_{2}=0.452, \mathrm{p}_{3}$ & $\begin{aligned} 3=1.000, p_{4} & =1 \\
p_{7} & =1\end{aligned}$ & $\begin{array}{l}1.000, p_{5}=0.47 \\
1.000\end{array}$ & $77, p_{6}=0.485$ & $\mathrm{p}_{8}=1.00$ & $0, p_{9}=1.000, p$ & $10=0.593$ \\
\hline Encephalocele & 0 & 2 & 2 & 4 & 2 & 0 & 2 & 2 & - & 4 \\
\hline $\mathbf{p}_{\mathrm{c}}$ & & 0.2 & 297 & & 0.1 & 115 & & & 0.179 & \\
\hline $\begin{array}{l}\text { Sig bet. } \\
\text { subgroups }\end{array}$ & & $\mathrm{p}_{1}=1$ & .000 & $p_{2}=0.396, p_{3}$ & $\begin{aligned}=0.045^{*}, p_{4} & =0 \\
p_{7} & =0\end{aligned}$ & $\begin{array}{l}0.404, p_{5}=0.45 \\
0.492\end{array}$ & $93, p_{6}=1.000$ & $\mathrm{p}_{8}=0.129$ & $9, p_{9}=1.000, p_{1}$ & $10^{0}=0.041^{*}$ \\
\hline
\end{tabular}

$\mathrm{p}_{\mathrm{c}}: \mathrm{p}$ value for comparing between control and each other groups

$\mathrm{p}_{1}$ : $\mathrm{p}$ value for comparing between Ila and IIb

$\mathrm{p}_{2}$ : $p$ value for comparing between IIIa and IIIb

$p_{3}: p$ value for comparing between IIIa and IIIc

$\mathrm{p}_{4}: p$ value for comparing between IIIa and IIId

$\mathrm{p}_{5}: \mathrm{p}$ value for comparing between IIIb and IIIc

$\mathrm{p}_{6}: \mathrm{p}$ value for comparing between IIIb and IIId

*: Statistically significant at $p \leq 0.05$

$\mathrm{p}_{7}$ : $p$ value for comparing between IIIc and IIId

$\mathrm{p}_{8}: \mathrm{p}$ value for comparing between IVa and IVb

$\mathrm{p}_{9}: \mathrm{p}$ value for comparing between IVa and IVc

$\mathrm{p}_{10}: \mathrm{p}$ value for comparing between IVb and IVc 
Table (5a): Skeletal anomalies in mice pups among studied groups.

\begin{tabular}{|c|c|c|c|c|c|c|c|c|c|c|}
\hline \multirow{3}{*}{ Skeletal abnormalities } & \multirow{3}{*}{$\begin{array}{l}\text { Group I } \\
\text { (control) (n } \\
=43 \text { ) }\end{array}$} & \multicolumn{2}{|c|}{ Group II (LCT) } & \multicolumn{4}{|c|}{ Group III (DBA) } & \multicolumn{3}{|c|}{ Group IV [18] } \\
\hline & & Ila $(n=42)$ & Ilb ( $n=39)$ & IIIa $(n=28)$ & IIIb $(n=34)$ & IIIc $(n=31)$ & IIId (n= 32) & Iva(n = 24) & $\operatorname{IVb}(n=42)$ & $\operatorname{IVc}(n=36)$ \\
\hline & & $\begin{array}{c}\text { (Nano } \\
10 \mathrm{mg} / \mathrm{kg})\end{array}$ & $\begin{array}{c}\text { (Nano } \\
20 \mathrm{mg} / \mathrm{kg})\end{array}$ & $\begin{array}{c}\text { (main } \\
10 \mathrm{mg} / \mathrm{kg})\end{array}$ & $\begin{array}{c}\text { (main } \\
25 \mathrm{mg} / \mathrm{kg})\end{array}$ & $\begin{array}{c}\text { (Nano } \\
10 \mathrm{mg} / \mathrm{kg})\end{array}$ & $\begin{array}{c}\text { (Nano } \\
25 \mathrm{mg} / \mathrm{kg})\end{array}$ & $\begin{array}{c}\text { (main } \\
10 \mathrm{mg} / \mathrm{kg})\end{array}$ & $\begin{array}{c}\text { (Nano } \\
10 \mathrm{mg} / \mathrm{kg})\end{array}$ & $\begin{array}{c}\text { (Nano } \\
50 \mathrm{mg} / \mathrm{kg})\end{array}$ \\
\hline \multicolumn{11}{|l|}{ In mice embryos } \\
\hline $\begin{array}{l}\text { Incomplete ossification of } \\
\text { nasal bone }\end{array}$ & 0 & 3 & 2 & 3 & 2 & 5 & 3 & 2 & 4 & 6 \\
\hline $\mathrm{p}_{\mathrm{c}}$ & & \multicolumn{2}{|c|}{0.162} & \multicolumn{4}{|c|}{$0.041^{*}$} & \multicolumn{3}{|c|}{$0.018^{*}$} \\
\hline Sig bet. Subgroups & & \multicolumn{2}{|c|}{$p_{1}=1.000$} & \multicolumn{4}{|c|}{$p_{2}=0.650, p_{3}=0.709, p_{4}=1.000, p_{5}=0.244, p_{6}=0.668$} & \multicolumn{3}{|c|}{$p_{8}=1.000, p_{9}=0.457, p_{10}=0.500$} \\
\hline $\begin{array}{l}\text { Incomplete ossification of } \\
\text { interparietal bone }\end{array}$ & 1 & 1 & 3 & 3 & 1 & 2 & 3 & 4 & 1 & 4 \\
\hline $\mathrm{p}_{\mathrm{c}}$ & & \multicolumn{2}{|c|}{0.658} & \multicolumn{4}{|c|}{0.455} & \multicolumn{3}{|c|}{0.282} \\
\hline Sig bet. Subgroups & & \multicolumn{2}{|c|}{$\mathrm{p}_{1}=0.347$} & \multicolumn{4}{|c|}{$p_{2}=0.320, p_{3}=0.661, p_{4}=1.000, p_{5}=0.602, p_{6}=0.348$} & \multicolumn{3}{|c|}{$\mathrm{p}_{8}=0.055, \mathrm{p}_{9}=0.702, \mathrm{p}_{10}=0.175$} \\
\hline \multicolumn{11}{|l|}{ Vertebrae } \\
\hline Open arch of atlas & 1 & 1 & 3 & 1 & 1 & 3 & 1 & 0 & 1 & 3 \\
\hline $\mathrm{p}_{\mathrm{c}}$ & & \multicolumn{2}{|c|}{0.658} & \multicolumn{4}{|c|}{0.679} & \multicolumn{3}{|c|}{1} \\
\hline Sig bet. Subgroups & & \multicolumn{2}{|c|}{$\mathrm{p}_{1}=0.347$} & \multicolumn{4}{|c|}{$p_{2}=1.000, p_{3}=0.614, p_{4}=1.000, p_{5}=0.341, p_{6}=1.000$} & \multicolumn{3}{|c|}{$p_{8}=1.000, p_{9}=0.268, p_{10}=0.330$} \\
\hline Incomplete ossification & 0 & 1 & 2 & 1 & - & 3 & 2 & 1 & 1 & 2 \\
\hline $\mathrm{p}_{\mathrm{c}}$ & & \multicolumn{2}{|c|}{0.551} & \multicolumn{4}{|c|}{0.34} & \multicolumn{3}{|c|}{0.319} \\
\hline Sig bet. Subgroups & & \multicolumn{2}{|c|}{$\mathrm{p}_{1}=0.606$} & $p_{2}=0.452, p_{3}$ & ${ }_{3}=0.614, p_{4}=1$. & $.000, p_{5}=0.10$ & $3, p_{6}=0.231$ & $\mathrm{p}_{8}=1.000$ & $p_{9}=1.000, p$ & ${ }_{10}=0.593$ \\
\hline Ribs & & & & & & & & & & \\
\hline Supernumerary & - & 2 & 2 & 3 & 1 & - & 1 & 1 & - & 2 \\
\hline $\mathrm{p}_{\mathrm{c}}$ & & 0.2 & & & 0.3 & 33 & & & 0.555 & \\
\hline Sig bet. Subgroups & & $\mathrm{p}_{1}=1$ & 000 & $p_{2}=0.320, p_{3}$ & ${ }_{3}=0.101, p_{4}=0$. & $.331, p_{5}=1.00$ & $0, p_{6}=1.000$ & $p_{8}=0.364$ & $p_{9}=1.000, p$ & ${ }_{10}=0.210$ \\
\hline Wavy ribs & - & 1 & 2 & 3 & 3 & 2 & 1 & 3 & 3 & 4 \\
\hline $\mathrm{p}_{\mathrm{c}}$ & & 0.5 & & & 0.1 & 114 & & & $0.034^{*}$ & \\
\hline Sig bet. Subgroups & & $\mathrm{p}_{1}=0$ & 606 & $\mathrm{p}_{2}=1.000, \mathrm{p}_{3}$ & $=0.661, p_{4}=0$ & $.331, p_{5}=1.00$ & $0, p_{6}=0.614$ & $\mathrm{p}_{8}=0.660$ & $p_{9}=1.000, p$ & ${ }_{10}=0.697$ \\
\hline Incomplete ossification & - & 1 & 2 & 1 & - & 2 & 1 & 1 & 1 & 1 \\
\hline $\mathrm{p}_{\mathrm{c}}$ & & 0.5 & & & 0.5 & 573 & & & 0.555 & \\
\hline Sig bet. Subgroups & & $\mathrm{p}_{1}=0$. & 606 & $p_{2}=0.452, p_{3}$ & $=1.000, p_{4}=1$ & $.000, p_{5}=0.22$ & $4, p_{6}=0.485$ & $p_{8}=1.000$ & $p_{9}=1.000, p$ & ${ }_{10}=1.000$ \\
\hline $\begin{array}{l}\text { Sternum incomplete } \\
\text { ossification }\end{array}$ & - & 1 & 2 & 1 & - & 1 & 1 & 1 & 1 & 2 \\
\hline $\mathrm{p}_{\mathrm{c}}$ & & 0.5 & & & 0.5 & 571 & & & 0.319 & \\
\hline Sig bet. Subgroups & & $\mathrm{p}_{1}=0$. & 606 & $p_{2}=0.452, p_{3}$ & $=1.000, p_{4}=1$ & $.000, p_{5}=0.47$ & $7, p_{6}=0.485$ & $p_{8}=1.000$ & $p_{9}=1.000, p$ & ${ }_{10}=0.593$ \\
\hline
\end{tabular}

$P_{c}: p$ value for comparing between control and each other groups

$p_{1}$ : $p$ value for comparing between IIa and IIb $p_{2}: p$ value for comparing between IIIa and IIIb $p_{3}: p$ value for comparing between IIIa and IIIc $p_{4}$ : $p$ value for comparing between IIIa and IIId $p_{5}$ : $p$ value for comparing between IIIb and IIIc $p_{6}$ : $p$ value for comparing between IIIb and IIId $p_{7}$ : $p$ value for comparing between IIIc and IIId $p_{8}$ : $p$ value for comparing between IVa and IVb $p_{9}$ : $p$ value for comparing between IVa and IV $\mathbf{c}$ $\mathrm{p}_{10}$ : $\mathrm{p}$ value for comparing between IVb and IVc *: Statistically significant at $\mathrm{p} \leq 0.05$

Table (5b): Skeletal anomalies in mice pups among studied groups.

\begin{tabular}{|c|c|c|c|c|c|c|c|c|c|c|c|}
\hline \multirow{3}{*}{$\begin{array}{c}\text { Skeletal } \\
\text { abnormalities }\end{array}$} & \multirow{3}{*}{$\begin{array}{l}\text { Group I } \\
\text { (control) (n } \\
=43)\end{array}$} & \multicolumn{2}{|c|}{ Group II (LCT) } & \multicolumn{4}{|c|}{ Group III (DBA) } & \multicolumn{3}{|c|}{ Group IV [18] } & \\
\hline & & Ila $(n=42)$ & llb ( $n=39$ ) & IIIIa $(n=28)$ & IIII $(n=34)$ & IIII $(n=31)$ & IIId ( $n=32)$ & Iva $(n=24)$ & $\mathrm{IVb}(\mathrm{n}=42)$ & $\operatorname{IVc}(n=36)$ & \\
\hline & & $\begin{array}{c}\text { (Nano } \\
10 \mathrm{mg} / \mathrm{kg})\end{array}$ & $\begin{array}{c}\text { (Nano } \\
20 \mathrm{mg} / \mathrm{kg})\end{array}$ & $\begin{array}{c}\text { (main } \\
10 \mathrm{mg} / \mathrm{kg})\end{array}$ & $\begin{array}{c}\text { (main } \\
25 \mathrm{mg} / \mathrm{kg})\end{array}$ & $\begin{array}{c}\text { (Nano } \\
10 \mathrm{mg} / \mathrm{kg})\end{array}$ & $\begin{array}{c}\text { (Nano } \\
25 \mathrm{mg} / \mathrm{kg})\end{array}$ & $\begin{array}{c}\text { (main } \\
10 \mathrm{mg} / \mathrm{kg})\end{array}$ & $\begin{array}{c}\text { (Nano } \\
10 \mathrm{mg} / \mathrm{kg})\end{array}$ & $\begin{array}{c}\text { (Nano } \\
50 \mathrm{mg} / \mathrm{kg})\end{array}$ & \\
\hline \multicolumn{12}{|l|}{ Fore limb } \\
\hline $\begin{array}{l}\text { Incomplete } \\
\text { ossification of } 5^{\text {th }} \\
\text { metacarpal }\end{array}$ & - & 2 & 3 & 2 & 2 & 3 & 2 & 3 & 4 & 5 & \\
\hline $\mathrm{p}_{\mathrm{c}}$ & & \multicolumn{2}{|c|}{0.162} & \multicolumn{4}{|c|}{0.114} & \multicolumn{3}{|c|}{$0.018^{*}$} & \\
\hline Sig bet. Subgroups & & \multicolumn{2}{|c|}{$\mathrm{p}_{1}=0.668$} & \multicolumn{4}{|c|}{$\begin{array}{c}p_{2}=1.000, p_{3}=1.000, p_{4}=1.000, p_{5}=0.663, p_{6}=1.000 \\
p_{7}=0.627\end{array}$} & \multicolumn{3}{|c|}{$p_{8}=0.699, p_{9}=1.000, p_{10}=0.725$} & \\
\hline $\begin{array}{l}\text { Incomplete } \\
\text { ossification of } \\
\text { phalanges }\end{array}$ & 1 & 2 & 4 & 3 & 1 & 4 & 3 & 2 & 3 & 7 & \\
\hline$p_{c}$ & & \multicolumn{2}{|c|}{0.42} & \multicolumn{4}{|c|}{0.3} & \multicolumn{3}{|c|}{0.109} & \\
\hline Sig bet. Subgroups & & \multicolumn{2}{|c|}{$\mathrm{p}_{1}=0.421$} & \multicolumn{4}{|c|}{$\begin{array}{c}\mathrm{p}_{2}=0.320, \mathrm{p}_{3}=1.000, \mathrm{p}_{4}=1.000, \mathrm{p}_{5}=0.184, \mathrm{p}_{6}=0.348 \\
\mathrm{p}_{7}=0.708\end{array}$} & \multicolumn{3}{|c|}{$p_{8}=1.000, p_{9}=0.293, p_{10}=0.173$} & *: Statistically significant at $p \leq 0.0$ \\
\hline \multicolumn{11}{|l|}{ Hind limb } & \\
\hline $\begin{array}{l}\text { Incomplete } \\
\text { ossification of } \\
\text { tarsus }\end{array}$ & 1 & 2 & 3 & 5 & 2 & 1 & 1 & 5 & 6 & 8 & \\
\hline$p_{c}$ & & \multicolumn{2}{|c|}{0.664} & \multicolumn{4}{|c|}{0.455} & \multicolumn{3}{|c|}{$0.009^{*}$} & \\
\hline Sig bet. Subgroups & & \multicolumn{2}{|c|}{$\mathrm{p}_{1}=0.668$} & \multicolumn{4}{|c|}{$\begin{array}{c}p_{2}=0.228, p_{3}=0.092, p_{4}=0.088, p_{5}=1.000, p_{6}=1.000 \\
p_{7}=1.000\end{array}$} & \multicolumn{3}{|c|}{$\mathrm{p}_{8}=0.511, \mathrm{p}_{9}=1.000, \mathrm{p}_{10}=0.391$} & \\
\hline $\begin{array}{l}\text { Incomplete } \\
\text { ossification of } \\
\text { phalanges }\end{array}$ & 1 & 2 & 2 & 3 & 2 & 1 & 4 & 1 & 1 & 6 & \\
\hline$p_{c}$ & & \multicolumn{2}{|c|}{0.658} & \multicolumn{4}{|c|}{0.293} & \multicolumn{3}{|c|}{0.281} & \\
\hline Sig bet. Subgroups & & \multicolumn{2}{|c|}{$\mathrm{p}_{1}=1.000$} & \multicolumn{4}{|c|}{$\begin{array}{c}\mathrm{p}_{2}=0.650, \mathrm{p}_{3}=0.337, \mathrm{p}_{4}=1.000, \mathrm{p}_{5}=1.000, \mathrm{p}_{6}=0.420 \\
\mathrm{p}_{7}=0.355\end{array}$} & \multicolumn{3}{|c|}{$p_{8}=1.000, p_{9}=0.225, p_{10}=0.044^{*}$} & \\
\hline
\end{tabular}

$P_{c}: p$ value for comparing between control and each other groups $p_{1}: p$ value for comparing between IIa and IIb

$p_{2}$ : $p$ value for comparing between IIIa and IIIb $p_{3}$ : $p$ value for comparing between IIIa and IIIc $p_{4}$ : $p$ value for comparing between IIIa and IIId $p_{5}$ : $p$ value for comparing between IIIb and IIIc $p_{6}$ : $p$ value for comparing between III b and IIId $p_{7}: p$ value for comparing between IIIc and IIId $\mathrm{p}_{8}$ : $p$ value for comparing between IVa and IVb $\mathrm{p}_{9}$ : $p$ value for comparing between IVa and IVc $\mathrm{p}_{10}$ : $p$ value for comparing between IVb and IVc 


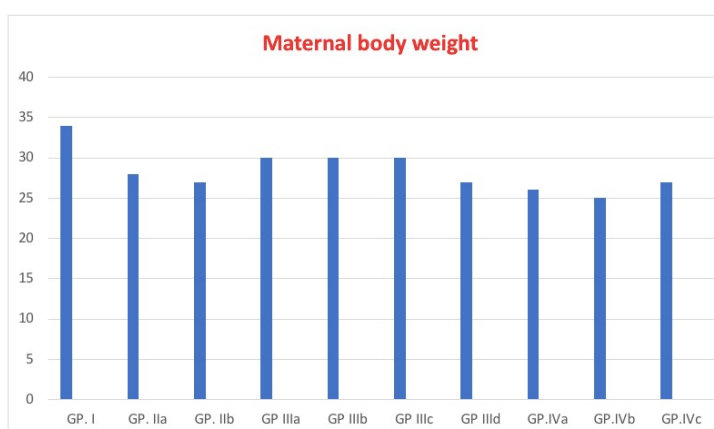

Fig. 1: A bar chart showing maternal body weight distribution among studied groups.

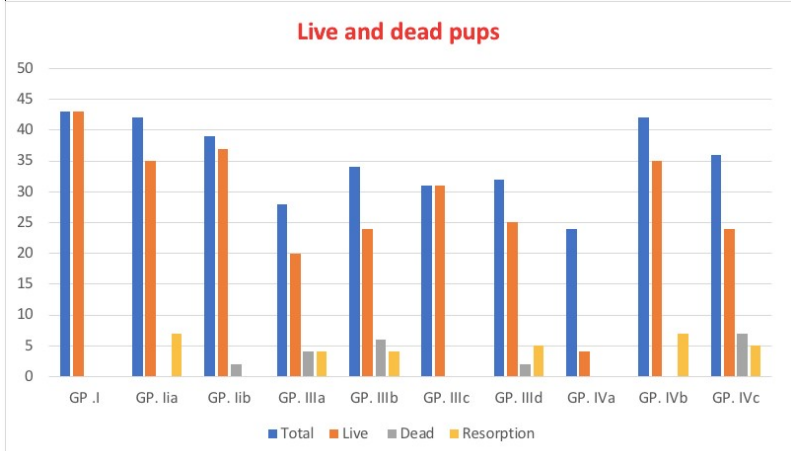

Fig. 2: A bar chart showing distribution of live and dead pups among studied groups.

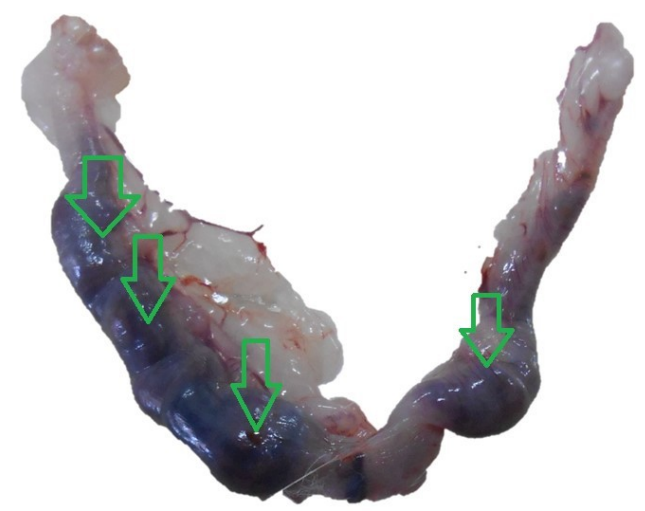

Fig. 3: A photograph of uterine horns of albino mice showing resorption sites (green arrows)
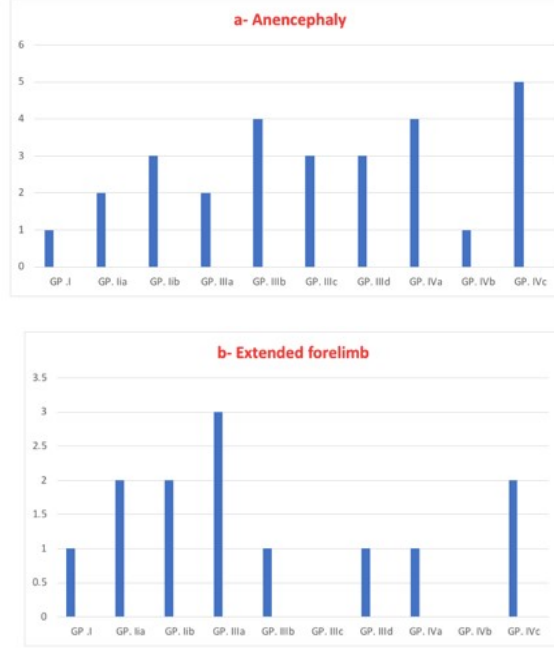

Fig. 4: Bar charts showing distrubition of :

a: Anencephally in mice pups among studied groups

b: Extended forelimb in mice pups among studied groups

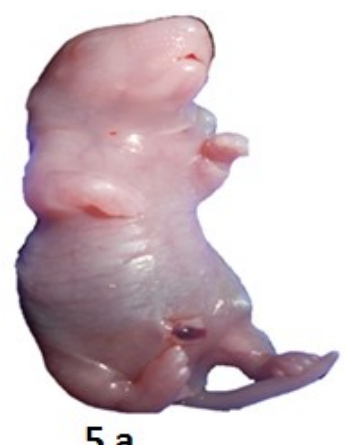

5 a

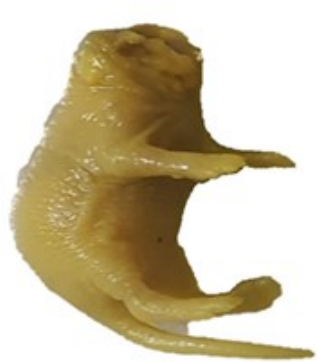

$5 \mathrm{~b}$

Fig. 5:

a: A Photograph showing normal mice pup.

b: A Photograph showing a mice pup with anencephally and extended forelimb (Bouin solution).

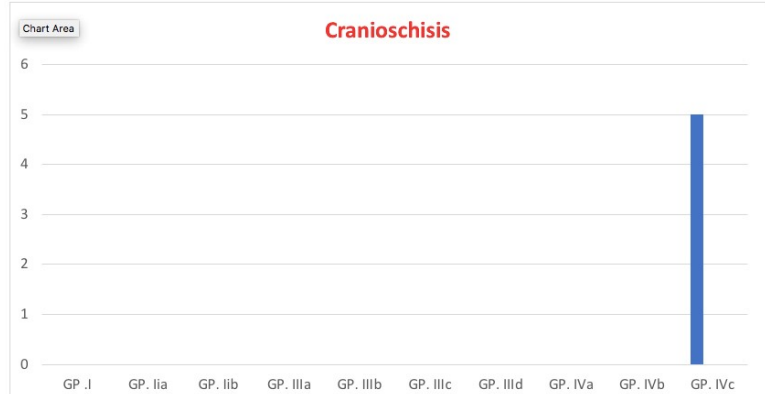

Fig. 6: A bar chart showing distribution of cranioschisis in mice pups among studied groups

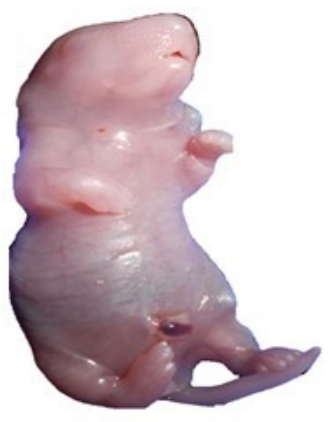

7 a

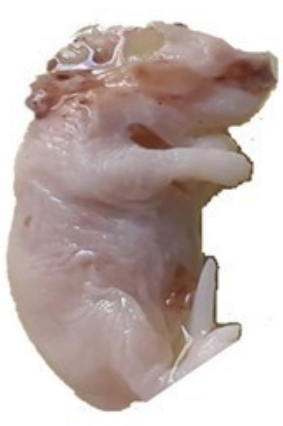

$7 \mathrm{~b}$
Fig. 7:

a: A Photograph showing a normal mice pup with normal skull vault.

b: A Photograph showing a mice pup with cranioschisis (incomplete skull vault).

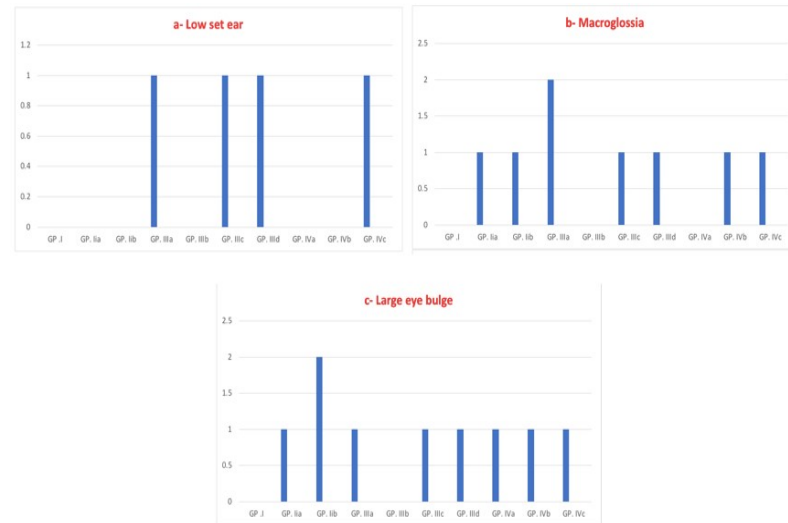

Fig. 8: Bar charts showing distrubition of :

a: Low set ear in mice pups of studied groups.

b: Macrglossia in mice pups of studied groups.

c: Large eye bulge in mice pups of studied groups. 

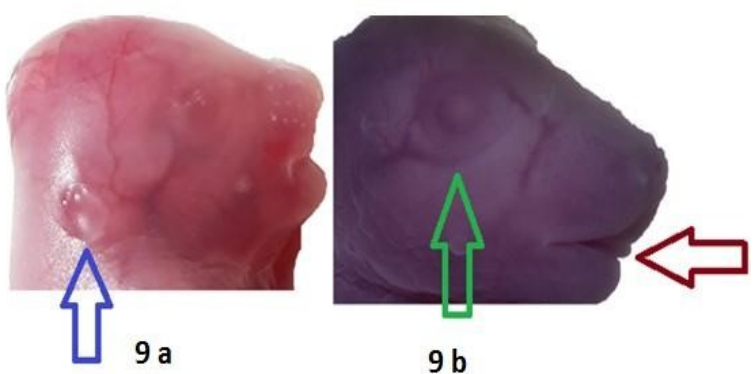

Fig. 9:

a: A photograph of a mice pup with a low set ear (blue arrow).

b: A photograph of a mice pup with macroglossia (red arrow) and a large eye bulge (green arrow).

$$
\text { Half body }
$$

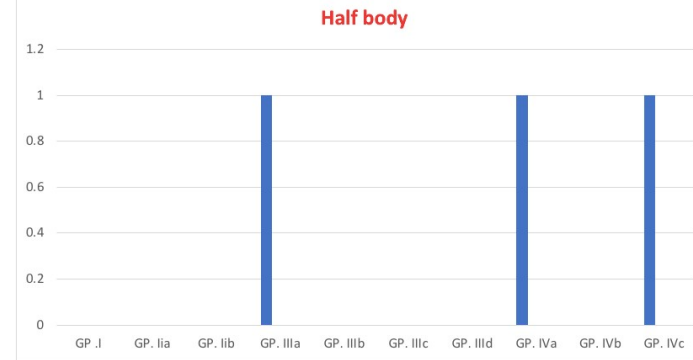

Fig. 10: A bar chart showing distribution of half body in mice pups among studied groups.

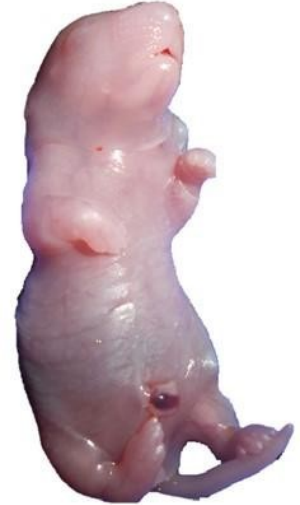

11 a

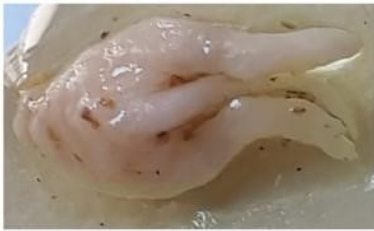

$11 \mathrm{~b}$
Fig. 11:

a: A Photograph of anterior view of a normal mice pup showing complete body.

b: A Photograph of a dorsal view of a mice pup displaying half body.

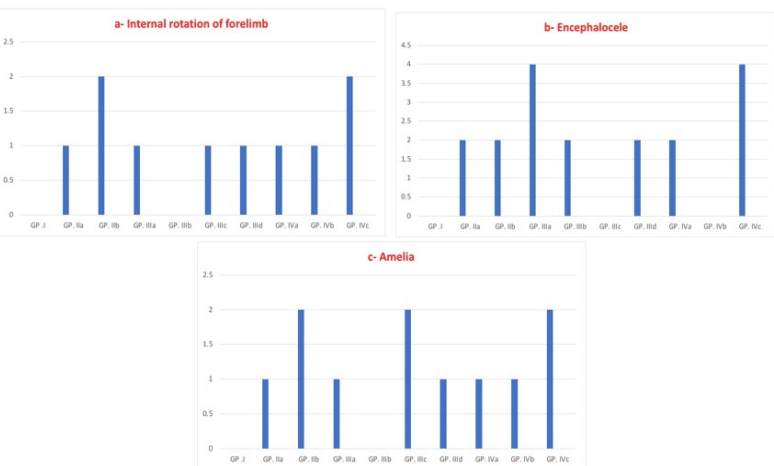

Fig. 12: Bar charts showing distrubition of:

a: Internal rotation of forelimb among studied groups.

b: Encephalocele among studied groups.

c: Amelia in mice pups among studied groups

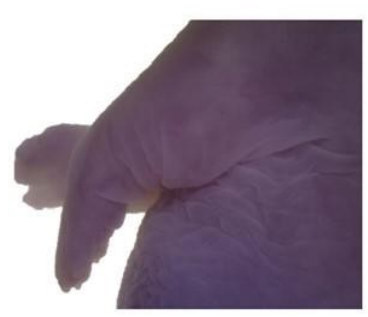

13 a

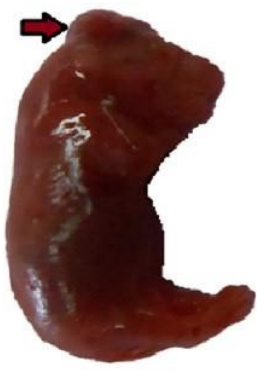

$13 \mathrm{~b}$
Fig. 13:

a: A Photograph of a mice pup showing internal rotation of left forelimb.

b: A Photograph of a mice pup showing encephalocele (red arrow) and amelia.

Incomplete ossification of nasal and interparietal bones

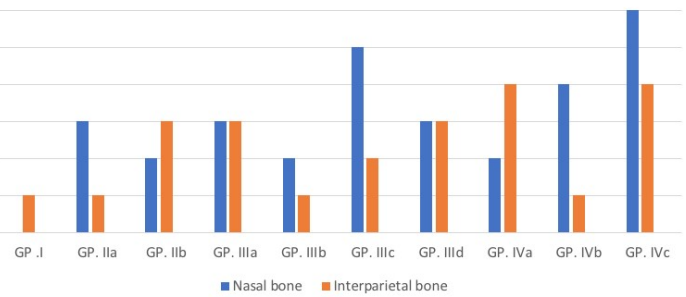

Fig. 14: A bar chart showing skull anomalies in mice pups among studied groups.

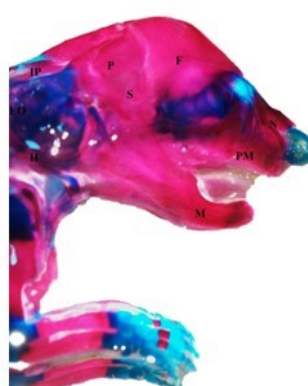

a

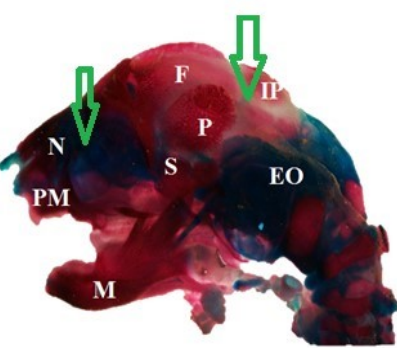

b
Fig. 15:

a: A photograph showing normal ossification of the nasal $(N)$, frontal $(F)$, parietal $(P)$, inter-parietal (IP), squamosal (S), mandible $(M)$, pre maxilla (PM), Exooccipital (EO), and hyoid $(\mathrm{H})$ bones.

b: A photograph showing incomplete ossification of nasal (N) and interparietal (IP) bones (green arrows).

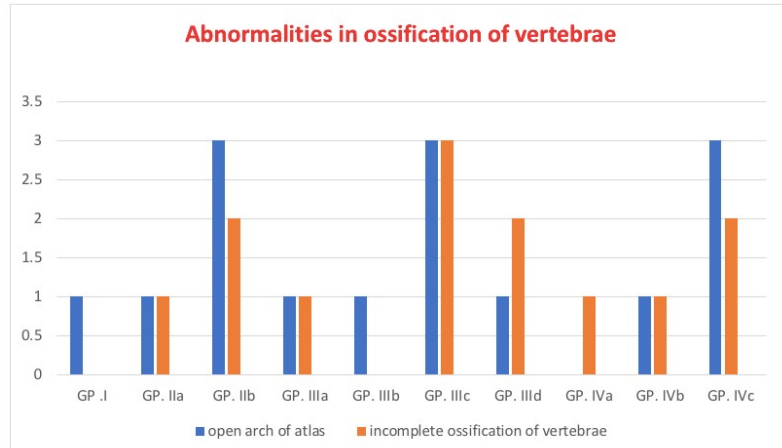

Fig. 16: A bar chart showing abnormalities in ossification of vertebrae in mice pups among studied groups. 


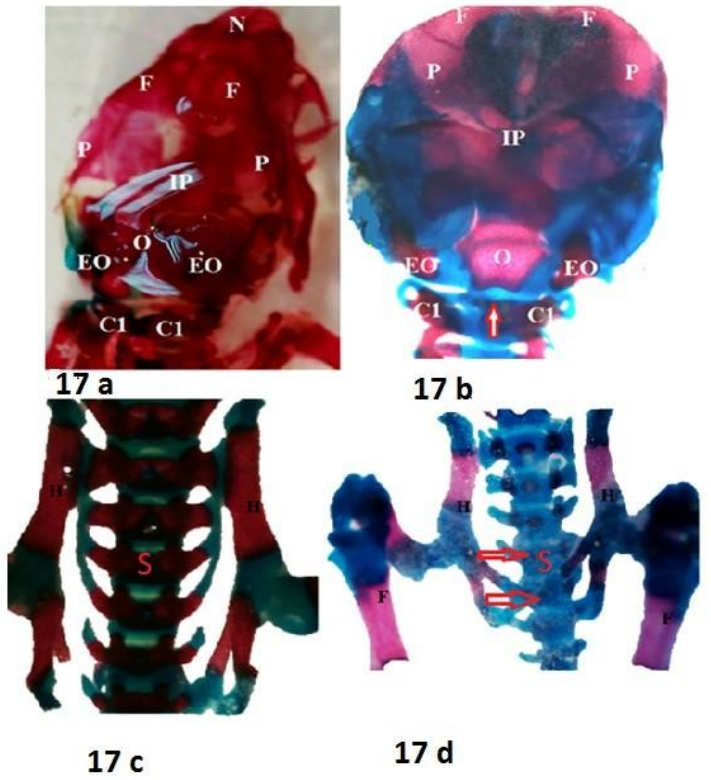

Fig. 17:

a: A photograph of a superior view of normal fetal skull, showing: nasal $(N)$, frontal $(F)$, parietal $(P)$, interparietal (IP) , occipital (O) , EO (Exoccipital) bones and normal closed arch of Atlas ( C1).

b: A photograph showing fetal skull with open arch of C1 (arrow).

c: A photograph showing normal ossification of hip bones $(\mathrm{H})$ and sacral vertebrae $(\mathrm{S})$.

d: A photograph showing incomplete ossification of sacral veretbrae (S, red arrows). note; $F$ : femur.

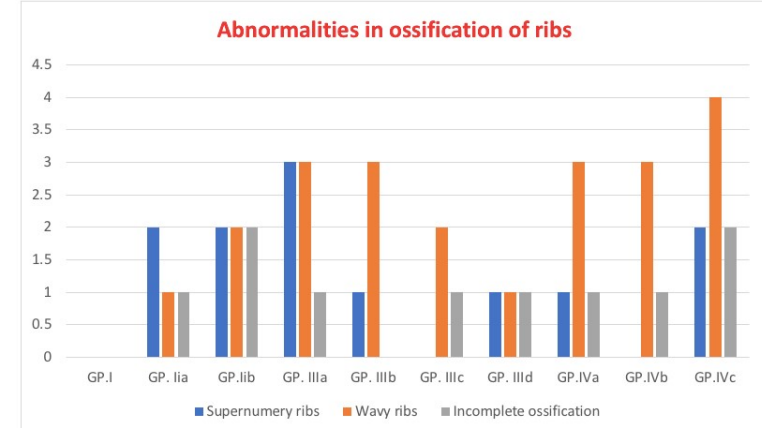

Fig. 18: A bar chart showing distribution of rib anomalies in mice puns among studied grouns.

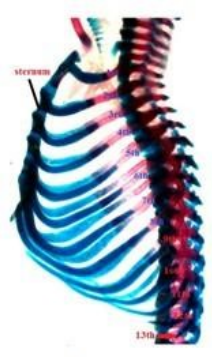

a

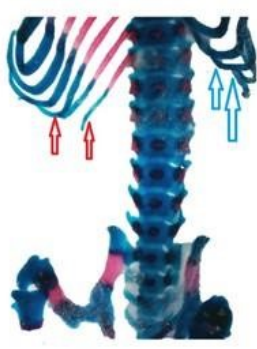

b

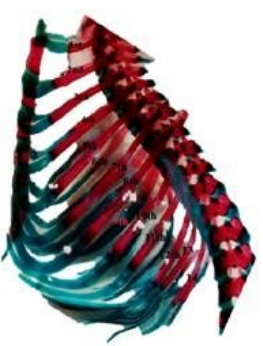

Fig. 19:

a: A photograph of a lateral view of normal fetal thoracic cage showing normal number, curve and ossification of ribs.

b: Aphotogaph of a ventral view of thoracic cage showing wavy lower ribs (red arrows) and incomplete ossification of ribs of the opposite side (blue arrows), c: Aphotogaph of a latral view of thoracic cage showing supernumerary ribs.
Abnormal ossification of sternum

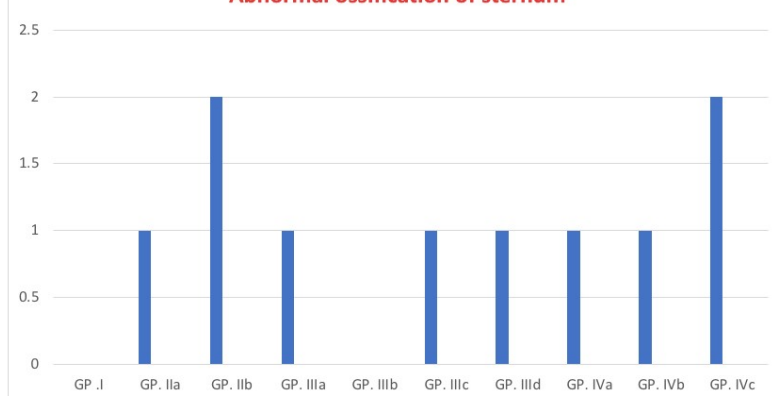

Fig. 20: A bar chart showing distribution of sternal anomalies in mice pups among studied groups.

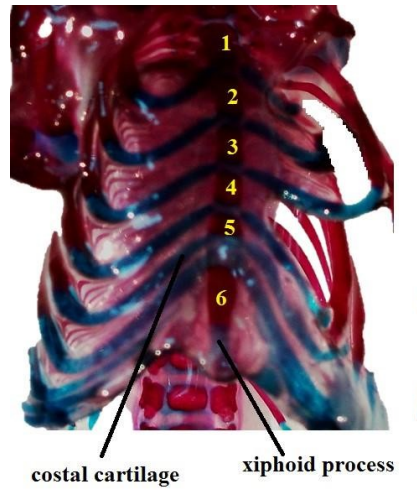

a

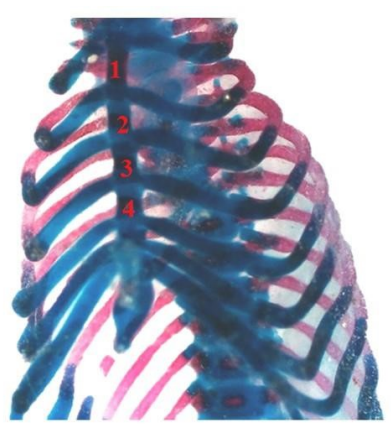

b
Fig. 21:

a: A photograph of a ventral view of thoracic cage showing a normal ossified sternum consisting of 6 sternebrae (1-6).

b: A photograph of a ventral view of thoracic cage showing unossified lower 2 sternebrae (sternabrae only 1-4).

Forelimb abnormalities

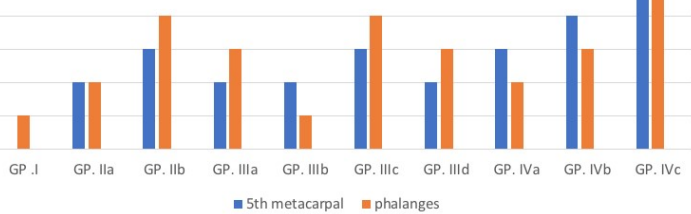

Fig. 22: A bar chart showing distribution of forelimb anomalies in mice pups among studied groups.
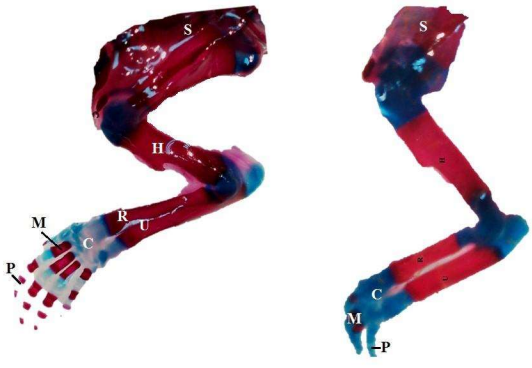

Fig. 23:

в

a: A photograph of normal fetal forelimb skeleton showing: scapula $(S)$, humerus $(H)$, radius $(R)$, ulna $(U)$, metacarpus (M), and phalanges (P). Note: carpus (C). b: A photograph showing incomplete ossification of 5th metacarpal $(\mathrm{M})$ and phalanges $(\mathrm{P})$. 
Hindlimb abnormalities

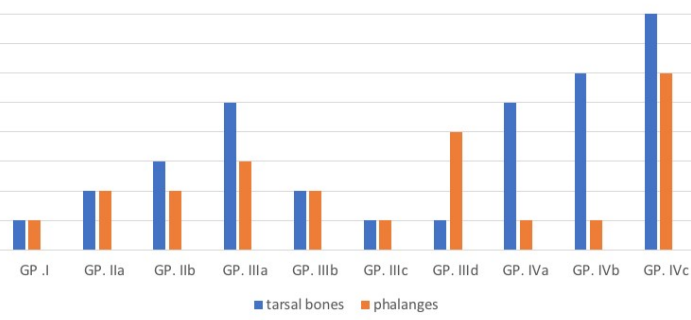

Fig. 24: A bar chart showing distribution of hindlimb anomalies in mice pups among studied groups.
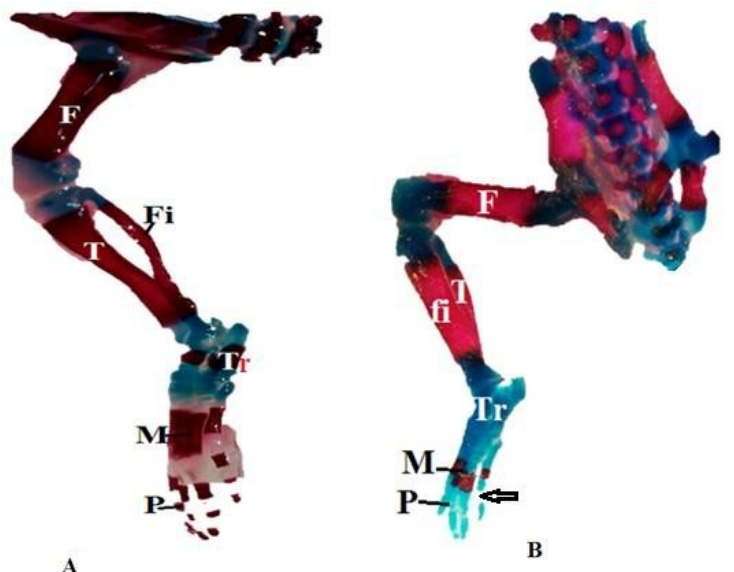

Fig. 25:

a: A photograph of normal fetal hindlimb skeleton showing normal ossification of: femur $(F)$, tibia $(T)$, fibula (Fi), tarsus (Tr), metatarsus(M), phalanges(P). b: A photograph of fetal hindlimb skeleton showing incomplete ossification of tarsus (Tr) and phalanges ( $P$, arrow).

(HD main $10 \mathrm{mg} / \mathrm{kg}$ ) and group Ivc (HD nano $50 \mathrm{mg} / \mathrm{kg}$ ) as compared to the control group (Table 4 , Figures 10, 11).

Amelia (absent limbs) was significantly increased in groups Ilb (LCT nano $20 \mathrm{mg} / \mathrm{kg}$ ) and IIIc (DBA nano 10mg / $\mathrm{kg}$ ) compared to the control group (Table 4 , Figures 12,13).

Rotational deformities of the extremities (Internal rotation of forelimb) was significantly increased in groups IIb (LCT nano $20 \mathrm{mg} / \mathrm{kg}$ ) and and group IVc (HD nano $50 \mathrm{mg} / \mathrm{kg}$ ) compared to the control group (Table 4 , Figures 12,13).

Encephalocele in group IIIa (DBA main $10 \mathrm{mg}$ $\mathrm{kg}$ ) and Ivc (HD nano 50mg / kg) was significantly increased compared to the control group (Table 4 , Figures 12, 13).

\section{DISCUSSION}

The widespread industrial use of pesticides induces environmental pollution and kills many beneficial non-target species. Several nano-particle pesticides provide an attractive solution to these problems with the small dissolvent that is required. This research was directed into the comparison between the main compounds and their nanoparticles.

In the present study, The total number of dead pups and resorption sites were increased significantly in groups (IIla (DBA main 10mg / $\mathrm{kg}$ ), IIIb (DBA main $25 \mathrm{mg} / \mathrm{kg}$ ) and Ivc (HD nano $50 \mathrm{mg} / \mathrm{kg}$ ) denoting their embryocidal effect.

Pal et al. [3]reported that nanoparticles are advantageous compared to the layer particles of the same elements for their high surface area / volume. Yet due to deeper penetration and high concentration this could be detrimental to other living organisms.

Meanwhile, Nishisaka et al. [8] studied the environmental effects of pesticides and nanopesticides by producing herbicide-containing paraquat nanoparticles and analyzing their potential genotoxic effects by using cytogenic test Allium Cepa treated with nanoparaquat, conventional paraquat, tripolyphosphate chitosan nanoparticles produced with or without hemic substances. In samples treated with nanoparaquat, the results showed less chromosome harm compared to conventional paraquat herbicide, a finding that is not in accordance with the present study.

In this study, Anencephally has been significantly increased in groups IIIb (DBA main $25 \mathrm{mg}$ / kg), Iva (HD main $10 \mathrm{mg} / \mathrm{kg}$ ) and Ivc (HD nano $50 \mathrm{mg} / \mathrm{kg}$ ), cranioschisis in group (HD nano $50 \mathrm{mg} / \mathrm{kg}$ ) and encephalocele in groups IIla (DBA main $10 \mathrm{mg} / \mathrm{kg}$ ) and Ivc (HD nano $50 \mathrm{mg} / \mathrm{kg}$ ) suggesting their teratogenic effect on the development of neural tube.

Yang et al.[20] investigated whether early gestational exposures to pesticides were associated with increased risk of anencephaly, spina bifida, cleft lip with or without cleft palate or cleft palate alone and found that there was no correlation between a variety of exposures to agricultural pesticides and the risk of identified birth defects.

Currently, limb abnormalities ranged from extended forelimb in group IIla (DBA main $10 \mathrm{mg} / \mathrm{kg}$ ), amelia in group IIb (LCT nano $20 \mathrm{mg}$ 
/ kg) and IIlc (DBA nano 10mg / kg) and internal forelimb rotation in group IIb (LCT nano $20 \mathrm{mg} / \mathrm{kg}$ ) and group Ivc (HD nano $50 \mathrm{mg} / \mathrm{kg}$ ) respectively.

Lin et al.[21] found in their research that cases of limb reduction defects with additional malformations appear to be associated with pesticide parental exposure. A finding that is in line with the present work.

In this study, macroglossia in groups IIIa (DBA main $10 \mathrm{mg} / \mathrm{kg}$ ) and Ivc (HD nano 50mg / kg), low set ears in groups IIla (DBA main 10mg / $\mathrm{kg}$ ), Illc (DBA nano 10mg / kg), Illd (DBA nano $25 \mathrm{mg} / \mathrm{kg}$ ) and Ivc (HD nano $50 \mathrm{mg} / \mathrm{kg}$ ) and large eye bulges in groups IIb (LCT nano $20 \mathrm{mg}$ $/ \mathrm{kg}$ ) and Ivc (HD nano 50mg / $\mathrm{kg}$ ) have been significantly increased.

Bhaskar et al. [22] identified a spectrum of eye defects in the form of microthalamus, anophthalmus, swelling and eye oedema, exophthalmus associated with chick embryos treated with deltamethrin. This observation is in line with the present finding in exophthalmos only. Malformations or abnormal development may be due to insecticide-induced gene mutation that is a strong inhibitor of cell proliferation, growth and differentiation and causes DNA fragmentation in the development of embryos. [23]

Zhang et al. [24] examined the effect of alumina nanoparticles (AINP) on neuro development and associated underlying mechanisms during pregnancy in female mice. The results showed that the aluminum content of the newborn hippocampus was significantly higher than the controls. They proposed that the underlying mechanism might be related to increased oxidative stress and a reduction in cerebral cortex neurotransmitter. They concluded that female mice exposure to AINP during pregnancy can induce offspring toxicity to neurodevelopment.

Brohi et al. [25] deduced that the toxicity of nanoparticles (NPs) in the reproductive system of animals is increasing and the entry of some NPs into both male and female reproductive organ, both directly in adult animals and in uters. In female animals, targeting of the uterus and ovaries for variety of NPs such as $\mathrm{TiO} 2, \mathrm{Cd}$ and Au. In males, NPs accumulate in the testis. Ag and TiO2 - based NPs appear to have dangerous effects, with an impact on cells in the seminiferous tubules, immune and inflammatory reactions, and sperm motility and morphology. Transplacental transfer of many types of NPs including $\mathrm{Au}, \mathrm{TiO} 2, \mathrm{SiO} 2$ and $\mathrm{C}$ is established in animals, which suggested the transfer of NPs to the vulnerable fetus, with varying toxic effects on the fetal brain and nerve development and future fertility.

With respect to skeletal anomalies in the present work, they ranged from incomplete ossification, open atlas arch, supernumerary, curved ribs, and incomplete ossification of phalanges and carpal or tarsal fore and hind limb bones. Most of these defects have been observed in group IVc (HD nano $50 \mathrm{mg} / \mathrm{kg}$ ) denoting higher skeletal ossification penetration, concentration, and impairment.

Yamashita, et al. [26] reported that nano particles can cross the placenta barrier in pregnant mice and cause neurotoxicity in their offspring. They showed that silica and titanium dioxide nanoparticles with diameters of $70 \mathrm{~nm}$ and $35 \mathrm{~nm}$, respectively, can cause pregnancy complications when injected intravenously into pregnant mice. These nanoparticles were found in the placenta, fetal liver and fetal brain. Mice treated with these nanoparticles had smaller uteri and smaller fetuses than untreated control.

Jokerst et al. [27] reviewed that some fundamental challenges humper nanoparticle (NP) development to the clinic which include uptake by the reticuloendothelial system (RES), in which NPs are rapidly shuttled out of circulation to the liver, spleen or bone marrow and nonspecific binding of NPs to nontargeted or non-diseased areas. The addition of PEG to the NP surface (PEGylation) can reduce many of these challenges. Yamashita et al. [26]reported that the recent studies have shown that nanoparticles can cross the placenta barrier in pregnant mice and cause neurotoxicity in their offspring. They exhibited that silica and titanium dioxide nanoparticles with diameters of $70 \mathrm{~nm}$ and 35 $\mathrm{nm}$, respectively can cause pregnancy complications when injected intravenously 
into pregnant mice. The silica and titanium dioxide nanoparticles were found in the placenta, fetal liver and fetal brain. Mice treated with these nanoparticles had smaller uteri and smaller fetuses than untreated controls.

Noori et al. [28] investigated the effect of magnetic iron oxide nanoparticles coated with dimercaptosuccinic acid on pregnancy and testicular development of mice by intraperitoneally injection to pregnant mice. The histological studies of the fetal liver and placenta sections showed presence of nanoparticles in these organ systems. The results showed a significant decrease in infants' growth from the mothers treated with doses higher than $50 \mathrm{mg} / \mathrm{kg}$. The testicular histological studs of these infants exhibited significantly decrease in spermatogonia, spermatocytes, spermatids and mature sperm.

Chauhan et al. [29] indicated that the antiproliferative effect of dibenzyl acetone in the intracellular amastigotes of leishmania donovani was more potent against intracellular amastigotes, the clinically more relevant stage of the parasite than its promastigotes stage. On comparison with reference drug miltefosine, dibenzalacetone showed IC50 value $(7.43 \mathrm{ug} / \mathrm{ml})$ closer to that reported for miltefosine (0.01-10.9 ug/ml). Dibenzalacetone -treated parasites showed remarkable morphological changes as ultrastructural alteration in the mitochondrion- kinetoplast complex, which showed intense mitochondrial swelling with an increase in the number of cristae, when compared to untreated cells.

A study on the analgesic and toxic properties of 6- dimethyl amino-4,4-diphenyl 3-heptanone hydrochloride (known as dolophine drug) has been made in some patients with pain due to advanced malignant disease [30]. Analgesic was obtained in patients to whom the drug was given sub cutaneously. Toxic reactions occurred with slight lethargy. Toxic manifestations were common when the drug received the drug by mouth such as anorexia, nausea, vomiting, dizziness, weakness or diaphoresis [31].

In metabolic studies, methyl-n-amyl ketone (2-heptanone) was detected in both serum Int J Anat Res 2021, 9(1.3):7912-27. ISSN 2321-4287 and urine from rats and monkeys exposed to both concentrations. In tissue distribution studies, carbon-14 labeled 2-heptanone was found accumulated in the liver, followed by kidney, pancreas and lungs. On the other hand, 2-heptanone can cause irritation of skin / eyes, respiratory system, headaches, vomiting and nausea (National library of Medicine (2019)[30].

Nanosuspensions are a special and commercial way of solving problems with hydrophobic drugs such as low solubility and poor bioavailability[7].

Due to various surface-to-volume changes, nanoparticles have the potential to influence biological processes, their potency increases. The binding of ligands or molecular coatings to the surface of nanoparticles enables the interaction and biocompatibility of nanoparticles-molecules. Intermolecular attractions between nanoparticles and biomolecular interactions will achieve this conjugation [32]. This may explain the higher skeletal defects observed in group Ivc (HD nano $50 \mathrm{mg} / \mathrm{kg}$ ).

Nano-encapsulation technologies enhance control of the condition where encapsulated pesticides are released into crop-built pesticides and manage the release of pesticides and their subsequent availability [33].

Therefore, the purpose of designing a polymer therapy is primarily to improve the potential of the appropriate drug by (1) increasing water solubility, especially relevant for certain drugs with lower aqueous solubility (2) stability toward degrading enzymes or diminished reticulo endothelial intake and (3) selective transfer of drugs to particular body sites of action [34].

On the other hand, because of its solubility in many organic solvents, PEG is also considered very hydrophobic, and the majority of PEGs used for drug modulation are either monomethoxy PEG or dihydroxy PEG.

The use of nanomaterials and technologies in the field of pesticides has made significant progress in recent years. Compounds of pesticide nanocapsules have slow release and defensive capacity due to their very small size, 
improvable droplet chemical reactivity of pesticides and target adsorption when spraying field which offer efficient and environmental benefits [35-37].

Bhuiyan et al. [38] Studied the effect of nanoparticles concentration and their sizes on the surface tension of nanofluids and reported that the surface tension of nanofluids increases with higher concentration-the surface tension of $\mathrm{Al} 2 \mathrm{O} 3, \mathrm{TiO}_{2}$ and $\mathrm{SiO} 2$ nanofluids varies from one another due to the variability of nanoparticles bulk density and higher nanoparticles surface tension than that of smaller nanoparticles.

Bhan et al. [39] tested the larvicidal efficacy of traditional (non-capsulated) insecticides (Temephos and Imidacloprid) and their nanoencapsulated formulations PEG was chosen for its biocompatibility and biodegradability, was used as a carrier and functions as insecticide-laden nano-dispersion capsules.

In this study melt-dispersion process has been used to prepare nanoparticles [10]. Specific PEG 6000 components were heated at $65^{\circ} \mathrm{C}$ separately. Various parts of LCT, DBA and HD were combined and stirred gently to these molten PEG. At room temperature, the mixtures were allowed to cool. Completely grounded in a mortar, the solidified mass was sieved using a 200 mesh sieve. The nanoparticles was prepared from the main compound according to certain equation.

A comparative toxicity assessment was performed by Amjad et al. [9] to evaluate the effect of cypermethrin nanoparticles (CYPNPS) and cypermethrin (CYP) on mural fish (Channa Punctatus). The results indicated that the percentage of micronuclei was increased significantly at different doses in CYP compared to CYPNPS. The increased levels of alkaline phosphatase, aspartate transaminase and alanine transaminase (ALT) in blood serum caused tissue impairment cellular damage in liver in the fish exposed to CYP compared to CYPNPs which indicated that CYPNPs is least toxic than the conventional from of CYP.

Pawar et al. [40] reported that the use of nanosuspentions in an universal formulation approach to increase the therapeutic performance of drugs in any route of administration. Nanosuspention is defined as very finely colloid biphasic, dispersed, solid drug particles in an aqueous vehicle, size below 1 um, without any matrix material, stabilized by surfactants and polymers, prepared by suitable methods for Drug Delivery applications, through various routs of administration like oral, topical, parenteral, ocular and pulmonary routs. So, the advantages of Nano suspentions are improved drug dispersibility and drug solubilization, increased therapeutic efficacy and reduced toxicity.

Sulaiman et al. [41] evaluated the biochemical effect of the oral administration of silver nanoparticles in Wister rats. Silver nanoparticles with 10,50 and $100 \mathrm{mg} / \mathrm{kg}$ body weight elevated the level of rat serum total cholesterol, triglyceride, free glycerol, low density lipoprotein - cholesterol and bilirubin when compared with the control. The level of high density lipoprotein- cholesterol was depleted by nanoparticles exposure. The levels of albumin, urea, creatinine as well as activities of aspartate transaminase and alkine phosphatase were decreased by nanoparticles. Furthermore, the nanoparticle treatment caused morphological lesions in rat cardiac, renal and hepatic tissues relative to the control.

Zhang et al. [42] investigated the potential impact of silver nanoparticles (AgNPs) on pregnant mice which administrated into pregnant mice via intravenous infusion at 1.0 $\mathrm{mg} / \mathrm{kg}$ doses at 6.5 days postcoitum (dpc); the pregnant mice were euthanized at 13.5, 15.5 and $17.5 \mathrm{dpc}$. The results showed that AgNPs exposure increased the meiotic progression of female germ cells in the fetal mouse ovaries, and maternal AgNPs exposure significantly disrupted imprinted gene expression in $15.5 \mathrm{dpc}$ embryos and placentas. AgNPs exposure significantly altered the DNA methylation levels of differentially methylated regions. Thus, the results indicated that early exposure to AgNPs has the potential to disrupt fetal and postnatal health through epigenetic changes in the embryo and abnormal development of the placenta. 
Chen et al. [43] studied sex differences in toxicity of polyethylene glycol-coated gold nanoparticles in mice. The GNPs caused significant increases in spleen and thymus indexes, which represented the activation of immune response, for female mice but not male mice. GNPs caused obvious increases of $W B C$ and $R B C$ in male mice but was not significant in female mice. It was found also that male mice suffered significant liver toxicity, with ALT and AST significantly increased but obvious kidney toxicity in female mice.

Despite the benefits of nanoparticles (NPs), various application have exposed humans and animals to their potential toxicities. The exposure of humans to NPs is concerned, they can enter the body through inhalation, ingestion, skin uptake, injection or implantation [44]. NP uptake could be intentional or non- intentional [45]. Thus, the wide use of nanomaterials has raised concerns about the negative impact of NPs on the reproductive systems of both men and women and on fetal health, particularly in view of the small size of NPs, their ease of penetration and biocompatibility and their potential ability to breach the placental barriers. Nanoparticles are associated with different disorders in animals, including pulmonary injury, hepatoxicity, immune - nanotoxicity, renal toxicity and irreversible testis damage [31].

Sardari et al [18]. assessed the toxic effects of size silver nanoparticles $(70 \mathrm{~nm})$ in rat, $\mathrm{s}$ kidney, liver and spleen tissues via oral gavage for 30 days which caused adverse impacts in dose-treated group (1 and $2 \mathrm{mg} /$ $\mathrm{kg}$ ) on liver, spleen and kidney. They suggested that these damages in the tissues may caused by the increase of free radicals and stimulation of oxidative stress.

Yang et al [46]. assessed the effect of gestational age and nanoparticle composition on fetal accumulation of maternally - administered nanomaterials in mice. They determined the placental and fetal uptake of $13 \mathrm{~nm}$ gold nanoparticles with different surface modifications (ferritin, PEG and citrate) following intravenous administration at embryonic day 5.5 - 15.5. They showed that prior to embryonic day 11.5 , all tested nanoparticles could be visualized and detected in fetal tissues in significant amount; however, fetal gold levels declined dramatically post embryonic day 11.5. So, less mature embryos are potentially more susceptible to nanoparticle toxicity. On the other hand, Au- nanoparticle accumulation in the extraembryonic tissues (EET) increased $6-15$ fold with gestational age. Fetal and EET accumulation of ferritin and PEG modified nanoparticles was greater than citrate - capped nanoparticles, which due to PEG coating conferring an increased rate of transfer.

\section{CONCLUSION}

Overviewing the results of the present study, it could be concluded that; Lambdacyhalothrin, dibenzalacetone and derivatives of heptanone result in numbers of congenital gross and skeletal abnormalities that indicate their teratogenic effect. nano-encapsulated compounds have a high penetration and teratogenic potential compared to the main compounds.

\section{Conflicts of Interests: None}

\section{REFERENCES}

[1]. Bakhta Aouey, M.D., Yassine Chtourou, Michèle Bouchard, and H.F. Abdelmajid Khabir, Pyrethroid insecticide lambda-cyhalothrin and its metabolites induce liver injury through the activation of oxidative stress and proinflammatory gene expression in rats following acute and subchronic exposure. Environ Sci Pollut Res 2017;24:15.

[2]. Hénault-Ethier, L., Health and environmental impacts of pyrethroid insecticides: What we know, what we don't know and what we should do about it - Executive summary and Scientific Literature Review. 2016.

[3]. Pal, S.L., et al., Nanoparticle: An overview of preparation and characterization. Journal of applied pharmaceutical science, 2011;1(6):228-234.

[4]. Qin, H., et al., Preparation and properties of lambdacyhalothrin/polyurethane drug-loaded nanoemulsions. RSC Advances, 2017;7(83):5268452693.

[5]. Amberg, N., et al., Effects of depilation methods on Imiquimod-induced skin inflammation in mice. Journal of Investigative Dermatology, 2017;137(2):528-531.

[6]. Alnotazy, M., et al., The possible protective effect of trimetazidine on imiquimod-induced psoriasis like skin inflammation in an animal model. International Journal of Research in Pharmaceutical Sciences, 2019;10:70-76. 
[7]. Patel, V.R. and Y.K. Agrawal, Nanosuspension: An approach to enhance solubility of drugs. J Adv Pharm Technol Res, 2011;2(2):81-7.

[8]. Nishisaka C, Grillo R, Sanches G, Fraceto L, Lima R. Analysis of the effects of pesticides and nanopesticides on the environment. InBMC proceedings 2014 Oct (Vol. 8, No. 4, pp. 1-1). BioMed Central.

[9]. Amjad, S., A.K. Sharma, and M. Serajuddin, Toxicity assessment of cypermethrin nanoparticles in Channa punctatus: Behavioural response, micronuclei induction and enzyme alteration. Regul Toxicol Pharmacol, 2018;100:127-133.

[10]. Desheesh, M.A., et al., Larvicidal Activity of NanoEncapsulated Lambda - Cyhalothrin Against Susceptible Mosquito Larvae (Culex Pipiens) in Comparison with Conventional form. Alexandria Science Exchange Journal, 2019;40: 568-573.

[11]. Shalgunov, V., et al., Comprehensive study of the drug delivery properties of poly(l-lactide)-poly(ethylene glycol) nanoparticles in rats and tumor-bearing mice. J Control Release, 2017; 261:31-42.

[12]. Tomar, M., A. Kumar, and S.K. Kataria, Evaluation of Acute toxicity of Lambda Cyhalothrin in Mus musculus L. Indian J Exp Biol, 2015;53(8):551-5.

[13]. Kim, S., et al., PubChem update: improved access to chemical data. Nucleic Acids Res, 2019;47(D1):D1102-D1109.

[14]. Malatesta, M., Histological and Histochemical Methods - Theory and Practice. European Journal of Histochemistry EJH, 2016;60(1):2639.

[15]. Schneider, S., Skeletal examination by double staining for ossified bone and cartilaginous tissue. Methods Mol Biol, 2013;947:215-21.

[16]. Kirkpatrick, L.A. and B.C. Feeney, A simple guide to IBM SPSS statistics for version 20.0. 2013, Belmont, Calif.: Wadsworth, Cengage Learning.

[17]. Kirkpatrick, L.A., A simple guide to IBM SPSS statistics : for version 20.0. 2013, [Belmont, Calif.]: Wadsworth Cengage Learning.

[18]. Sardari, R.R.R., et al., Toxicological effects of silver nanoparticles in rats. African Journal of Microbiology Research, 2012;6(27):5587-5593.

[19]. Makris, S.L., et al., Terminology of developmental abnormalities in common laboratory mammals (version 2). Congenit Anom (Kyoto), 2009;49(3):123-246.

[20]. Yang, W., et al., Residential agricultural pesticide exposures and risk of neural tube defects and orofacial clefts among offspring in the San Joaquin Valley of California. Am J Epidemiol, 2014;179(6):740-8.

[21]. Lin, S., E.G. Marshall, and G.K. Davidson, Potential parental exposure to pesticides and limb reduction defects. Scand J Work Environ Health, 1994;20(3):166-79.

[22]. Bhaskar, N., et al., Effect of deltamethrin containing formulation on developing chick embryo: Morphological and skeletal changes. International Journal of Toxicological and Pharmacological Research, 2012;4:81-87.
[23]. Mobarak, Y.M. and M.A. Al-Asmari, Endosulfan impacts on the developing chick embryos: Morphological, morphometric and skeletal changes. International Journal of Zoological Research, 2011;7(2):107-127.

[24]. Zhang, Q., et al., Exposure to Alumina Nanoparticles in Female Mice During Pregnancy Induces Neurodevelopmental Toxicity in the Offspring. Front Pharmacol, 2018;9:253.

[25]. Brohi, R.D., et al., Toxicity of Nanoparticles on the Reproductive System in Animal Models: A Review. Front Pharmacol, 2017;8:606.

[26]. Yamashita, K., et al., Silica and titanium dioxide nanoparticles cause pregnancy complications in mice. Nat Nanotechnol, 2011;6(5):321-8.

[27]. Jokerst, J.V., et al., Nanoparticle PEGylation for imaging and therapy. Nanomedicine (Lond), 2011;6(4):715-28.

[28].Noori, A., et al., Effect of magnetic iron oxide nanoparticles on pregnancy and testicular development of mice. African Journal of Biotechnology, 2011;10(7):1221-1227.

[29]. Chauhan, I.S., et al., Chemoprevention of Leishmaniasis: In-vitro antiparasitic activity of dibenzalacetone, a synthetic curcumin analog leads to apoptotic cell death in Leishmania donovani. Parasitol Int, 2018;67(5):627-636.

[30]. Gutiérrez-García, A.G., C.M. Contreras, and R. Mendoza-López, 2-Heptanone Produces SensorialEmotional Changes, Depending on Length of Exposure. Revista Costarricense de Psicología, 2015;34:04-17.

[31]. Vance, M.E., et al., Nanotechnology in the real world: Redeveloping the nanomaterial consumer products inventory. Beilstein J Nanotechnol, 2015;6(1):1769-80.

[32]. Salata, O., Applications of nanoparticles in biology and medicine. J Nanobiotechnology, 2004;2(1):3.

[33]. Eerikäinen, H., et al., Aerosol flow reactor method for synthesis of drug nanoparticles. European Journal of Pharmaceutics and Biopharmaceutics, 2003;55(3):357-360.

[34]. Knop, K., et al., Poly (ethylene glycol) in drug delivery: pros and cons as well as potential alternatives. Angewandte chemie international edition, 2010;49(36):6288-6308.

[35]. Khot, L.R., et al., Applications of nanomaterials in agricultural production and crop protection: a review. Crop protection, 2012;35:64-70.

[36]. Nair, R., et al., Nanoparticulate material delivery to plants. Plant science, 2010;179(3):154-163.

[37]. Tebaldi, M.L., R.M. Belardi, and S.R. Montoro, Polymers with Nano-encapsulated Functional Polymers. Design and Applications of Nanostructured Polymer Blends and Nanocomposite Systems; Elsevier: Amsterdam, The Netherlands, 2016.

[38]. Bhuiyan, M.H.U., et al., Effect of nanoparticles concentration and their sizes on surface tension of nanofluids. Procedia Engineering, 2015;105: 431-437. 
[39]. Bhan, S., L. Mohan, and C.N. Srivastava, Relative larvicidal potentiality of nano-encapsulated Temephos and Imidacloprid against Culex quinquefasciatus. Journal of Asia-Pacific Entomology, 2014;17(4):787-791.

[40]. Pawar, S.S., et al., Nanosuspension technologies for delivery of drugs. J Nanosci Nanotechnol, 2017;4:59-66.

[41]. Sulaiman, F.A., et al., Biochemical and morphological alterations caused by silver nanoparticles in Wistar rats. Journal of Acute Medicine, 2015;5(4):96-102.

[42]. Zhang, X.F., et al., Silver nanoparticles cause complications in pregnant mice. Int J Nanomedicine, 2015;10:7057-71.

[43]. Chen, J., et al., Sex differences in the toxicity of polyethylene glycol-coated gold nanoparticles in mice. Int J Nanomedicine, 2013;8:2409-19.
[44]. Oberdörster, G., E. Oberdörster, and J. Oberdörster, Nanotoxicology: an emerging discipline evolving from studies of ultrafine particles. Environ Health Perspect 2005;113:823-839.

[45]. Yah, C.S., S.E. Iyuke, and G.S. Simate, A review of nanoparticles toxicity and their routes of exposures. Iranian Journal of Pharmaceutical Sciences, 2012;8(1):299-314.

[46]. Yang, H., et al., Effects of gestational age and surface modification on materno-fetal transfer of nanoparticles in murine pregnancy. Sci Rep, 2012;2:847.

How to cite this article: El Sayed Aly Mohammed Metwally, Mohammed Abdelfattah Desheesh, Doaa Mohammed Abd El-kawy Elmasry, Mahmoud Morshedy Farg, Hamada Mahmoud Youssef, Rasha M. Elshinety. Congenital skeletal malformations induced by lambda-cyhalothrin, dibenzalacetone and heptanone derivatives in embryos of albino mice. Int J Anat Res 2021;9(1.3):7912-7927. DOI: 10.16965/ijar.2021.109 